\title{
Study of the nonlocal active sound control with preservation of desired field in time domain
}

DOI:

$10.1121 / 10.0002880$

$10.1121 / 10.0002880$

Document Version

Accepted author manuscript

Link to publication record in Manchester Research Explorer

\section{Citation for published version (APA):}

Zhou, C., \& Utyuzhnikov, S. (2020). Study of the nonlocal active sound control with preservation of desired field in time domain. Journal of the Acoustical Society of America, 148(6), 3886-3899. https://doi.org/10.1121/10.0002880, https://doi.org/10.1121/10.0002880

\section{Published in:}

Journal of the Acoustical Society of America

\section{Citing this paper}

Please note that where the full-text provided on Manchester Research Explorer is the Author Accepted Manuscript or Proof version this may differ from the final Published version. If citing, it is advised that you check and use the publisher's definitive version.

\section{General rights}

Copyright and moral rights for the publications made accessible in the Research Explorer are retained by the authors and/or other copyright owners and it is a condition of accessing publications that users recognise and abide by the legal requirements associated with these rights.

\section{Takedown policy}

If you believe that this document breaches copyright please refer to the University of Manchester's Takedown Procedures [http://man.ac.uk/04Y6Bo] or contact uml.scholarlycommunications@manchester.ac.uk providing relevant details, so we can investigate your claim.

\section{OPEN ACCESS}


Study of the nonlocal active sound control with preservation of desired field in time domain

Chuxiong Zhou ${ }^{1}$ and Sergey Utyuzhnikov ${ }^{1, a)}$

Department of Mechanical, Aerospace \& Civil Engineering, University of Manchester, Manchester, M13 9PL, UK

(Dated: 22 October 2020) 
In the active sound control, a domain is protected from externally generated noise via constructing secondary sound sources, which are called controls. These controls are applied on the boundary of the shielded domain. Apart from the external noise, a desired sound generated by interior sources should also be retained inside the shielded domain. However, it turns out it is a challenge to preserve the internally generated sound unaffected due to both the reverse effect of the controls on the input data and their sparse distribution on the boundary. To take into account the reverse effect, an innovative algorithm based on nonlocal control is implemented in the time domain for the first time. Its real-time practical implementation may include preliminary tuning to the real surrounding conditions. A number of test cases are considered including external broadband noise and internal monochromatic desired sound. A sensitivity analysis is carried out with respect to some key design parameters such as density of sensors and controls as well as their respective geometrical displacement from one another determined by the Hausdorff distance. It is demonstrated that the nonlocal control provides the noise attenuation level which is not much sensitive to the presence of the desired sound.

a) S.utyuzhnikov@manchester.ac.uk; Also at: Moscow Institute of Physics \& Technology, 141700, Russia. 


\section{INTRODUCTION}

With more and more stringent noise control regulations in industry, high interest has been drawn to noise control during past few decades. The passive noise attenuation is achieved via implementation of insulation materials and can be quite efficient in application to bounded domains to be protected from high frequency noise. Meanwhile, the application of passive noise control becomes problematic if a low frequency noise propagates since long waves can decay much slower than short waves (Hansen and Snyder, 1996). In turn, the active sound control (ASC) has proved to be efficient for the attenuation of low-frequency noise (Canevet, 1978). In the ASC problems, a domain, which is usually bounded, is supposed to be shielded from noise generated outside by constructing secondary sound sources also known as controls. In the classical formulation, the ASC is based on the application of Huygens' principle (Baker and Copson, 1939). As a result, an anti-noise is generated by controls positioned at the perimeter of a domain to be shielded. In contrast to the passive sound control, ASC does not use any absorbing material to cancel noise. Practically, passive sound control is mostly applicable to the attenuation of mid- and high-frequency noise, whereas the ASC is better suitable for low frequency noise due to the space and time limitations on the control system (Elliott and Nelson, 1990). Therefore, the ASC can be regarded as an effective supplement to the passive sound control.

The conventional ASC control systems are based on one of the two principal algorithms with feed-back and feed-forward controls (Elliott, 2001; Elliott and Sutton, 1996; Nelson and Elliott, 1991). In the feed-back system, the noise signal is detected by a set of error sensors 
implemented inside the shielded domain. The operation of feed-forward control is based on a predetermined strategy that the measurement of noise is completed before the controls (Kletschkowski, 2011). To realize the noise attenuation by the action of controls, the filtered$\mathrm{x}$ least mean square (FxLMS) algorithm is commonly used for feed-back and feed-forward systems to adjust the noise signal (Hansen et al., 2012). Some examples of current ASC research include headphones (Hansen et al., 2012), ventilation and refrigeration systems (Tokhi et al., 2002), aircrafts, elevators and car cabins (Bai and Chen, 2000; Broadbent, 1976; Landaluze et al., 2003). In these applications the controls are implemented over the entire acoustically transparent boundary to be shielded. However, in some applications the controls can be applied only on a part of the boundary of a protected domain. For example, in (Zou et al., 2010) the controls were mounted on the top of anti-noise barriers to attenuate noise from outdoor transformers.

It should be noted that the preservation of desired sound is usually not considered in the conventional ASC problems (Williams, 1984). In practice, especially for a feed-back system, it is problematic to differentiate immediately between noise and desired sound in the total acoustic field from the primary sources. It seems that for the first time the ASC problem with the existence of an interior component was analysed by Malyuzhinets in (Malyuzhinets, 1971). He obtained the solution to the ASC problem in a bounded domain for the case when the total field from only primary sources is available. This solution presumes a continuous distribution of the controls at the perimeter of the domain to be shielded. A discrete realization of this approach, which was more practical, was made in paper (Konyaev et al., 1977). 
Ryaben'kii obtained a solution to the stationary problem in a quite general formulation in discrete spaces (Ryabenkii, 1995, 2002). Similar to (Malyuzhinets, 1971), this solution presumes the knowledge of the primary field at the perimeter of the domain to be shielded. Thanks to a discrete form, it can be realized via a set of monopoles and dipoles. This opportunity was clearly demonstrated by Tsynkov (Tsynkov, 2003). In his paper the links between finite-difference surface potentials, solution from (Ryabenkii, 2002) and acoustic equations are shown. The developed technique is applied to the Helmholtz equations in (Loncaric et al., 2001) where a link with the surface Calderón potentials was first provided. For the first time, the problem on ASC was considered in composite domains in (Ryaben'kii et al., 2007).

The approach described above was extended to unsteady problems in (Ryaben'kii and Utyuzhnikov, 2006a, 2007, 2006b; Utyuzhnikov, 2009a, 2010a). In (Utyuzhnikov, 2010a), the operation of ASC was explicitly demonstrated via the exact analytical solution to the unsteady problem obtained in a quite general formulation. A desired sound was allowed to be present in the shielded domain. It was shown that ASC can be realized even for resonance regimes in bounded domains. Afterwards, Utyuzhnikov was the first who considered the solvability of the nonlinear problem via the apparatus of generalized nonlinear potentials (Utyuzhnikov, 2010b) introduced. The experimental realization of the potential-based approach applied to ASC was addressed in a number of publications (see, e.g.,(Lim et al., 2014, 2011, 2009; Ntumy and Utyuzhnikov, 2014, 2015)).

It is to be noted the key assumption for the control mentioned above is based on an apriori knowledge of the primary field generated by both undesired and desired sources. 
This is hardly achievable in practice since in real-time realization the sound component generated by the controls is inevitable in the input data to be measured. As shown by Ryaben'kii and Utyuzhnikov, controls can double the desired sound outside the protected region (Ryaben'kii and Utyuzhnikov, 2007, 2006b). This makes the original field from the primary sources unattainable. In some practical applications, mostly related to the noise control in ventilation, this problem is partially resolved via directional measurements (see, e.g., (Huang et al., 2011; Jakob and Moser, 2003a,b; Kwon and Park, 2011; Sieck and Lau, 2011; Wang et al., 2016)). In (Ryaben'kii, 2011), it is proposed to solve an auxiliary local initial-boundary value problem across the control boundary to generate the control function. Although in a finite-difference formulation considered in the paper the algorithm is strict, its practical realization is very problematic. Indeed, it presumes a second-order numerical differentiation of noisy and contaminated input data. Moreover, for the algorithm it is important that the controls are supposed to be acoustically transparent and have no volume that is practically not realizable.

A new approach to tackle this problem was proposed by Utyuzhnikov in (Utyuzhnikov, 2009b) and (Utyuzhnikov, 2014). In these papers the proposed control is nonlocal. This means the operation of a control depends on the field along an entire control surface. The approach is based on the projection property of Calderón surface potentials. As a result, the contribution of the desired sound and secondary field is effectively subtracted from the total field. In this way, there is no need to predict desired sound thanks to the automatic separation of noise and desired sound from the total field. A practical algorithm for realization of the nonlocal active control was proposed in (Utyuzhnikov, 2017). The algorithm 
is potentially applicable to realization in real-time regimes. It was implemented for the first time in the frequency domain by Zhou and Utyuzhnikov in (Zhou and Utyuzhnikov, 2020). In that paper it was demonstrated that the nonlocal control has an advantage over the local control even without the presence of desired sound.

The current paper is organized as follows. First, an introduction to the general formulation of ASC problem is provided in the next section. It presumes the presence of desired sound along with noise generated outside a protected area. Then, Section III addresses the local and nonlocal ASC algorithms. The nonlocal control is based on a potential-based algorithm. The problem of the reverse effect of the controls on the input data is discussed. The results of numerical experiments carried out for a few pure-tone sources are demonstrated in Section IV. A sensitivity analysis is considered with respect to different input parameters such as the distribution density of sensors and dynamics as well as their relative geometrical displacement. Finally, the test cases with a broadband noise are discussed in Section V which is followed by the Conclusion.

\section{GENERAL FORMULATION OF THE ASC PROBLEM}

We consider the following problem formulation. Introduce some domain $D_{0}: D_{0} \subseteq R^{3}$. In addition, we introduce subdomain $D^{+}: D^{+} \subset D_{0}$ with smooth enough boundary $\Gamma$. This subdomain is supposed to be shielded from the noise generated in domain $D^{-}$: $=D_{0} \backslash \overline{D^{+}}$. 
JASA

Assume the acoustic field in domain $D_{0}$ is described by the following linear initialboundary-value problem (IBVP):

$$
\begin{aligned}
& p_{t t}-a^{2} \Delta p=f, \\
& p(\boldsymbol{x}, 0)=p_{t}(\boldsymbol{x}, 0)=0, \quad \boldsymbol{x} \in D_{0}, \\
& p \in \Xi_{D_{0}},
\end{aligned}
$$

where $p$ is the sound pressure; $a$ is the speed of sound; $f$ corresponds to the acoustic sources; $\Xi_{D_{0}}$ is a functional space such that the solution of IBVP (1) and (2) exists and is unique. If domain $D_{0}$ is unbounded, then space $\Xi_{D_{0}}$ is determined by the Sommerfeld boundary conditions.

The acoustic source $f$ presented by the right hand side in (1) is composed of noise sources $f^{-}$and desired sound sources $f^{+}$:

$$
\begin{aligned}
& f=f^{+}+f^{-}, \\
& \text {supp } f^{+} \subset D^{+}, \\
& \text {supp } f^{-} \subset D^{-} .
\end{aligned}
$$

In turn, the total sound field $p$ consists of desired sound $p^{+}$and noise $p^{-}$:

$$
p=p^{+}+p^{-}
$$

Assume that we are going to protect domain $D^{+}$from noise $p^{-}$. At the same time, it is required to retain the desired field $p^{+}$unaffected there. It is worth noting that $p^{+}$also includes any field reflected from the boundaries if it exists. The same definition is applicable 
to $p^{-}$. Then, the ASC becomes an inverse source problem: find secondary sound sources $G_{0}$ on boundary surface $\Gamma$ such that the solution to IBVP

$$
\begin{aligned}
& p_{c \mid t t}-a^{2} \Delta p_{c}=f+G_{0} \\
& \operatorname{supp} G_{0} \subset D \backslash D^{+}, \\
& p_{c}(\boldsymbol{x}, 0)=p_{c \mid t}(\boldsymbol{x}, 0)=0, \quad \boldsymbol{x} \in D_{0}, \\
& p_{c} \in \Xi_{D_{0}} .
\end{aligned}
$$

coincides with $p^{+}$in $D^{+}: p_{c}=p^{+}$.

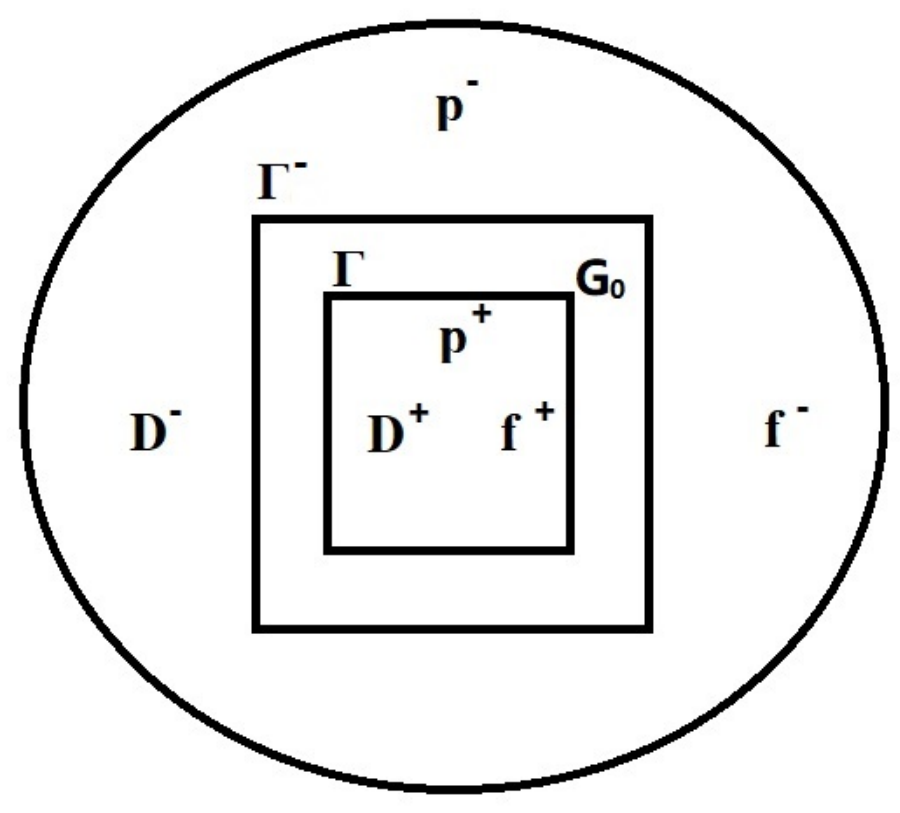

FIG. 1. Domain sketch. 


\section{ACTIVE SOUND CONTROL}

\section{A. Local sound control}

Consider the control function as a linear combination of simple and double-layer sources:

$$
G_{0}(\Gamma, t)=-a^{2} \delta(\Gamma) \frac{\partial p}{\partial \boldsymbol{n}} \mid \Gamma-a^{2} p_{\Gamma} \frac{\partial \delta(\Gamma)}{\partial \boldsymbol{n}}
$$

where $\boldsymbol{n}$ is the outward normal to boundary $\Gamma ; \delta(\Gamma)$ represents the surface delta-function associated with $\Gamma ; p_{\Gamma}$ is the restriction of $p$ to $\Gamma: p_{\Gamma}=p(\Gamma, t) ; p(\Gamma, t)$ corresponds to the total field from the primary sources at boundary $\Gamma$.

Source $G_{0}$ generates field $p_{l}$ :

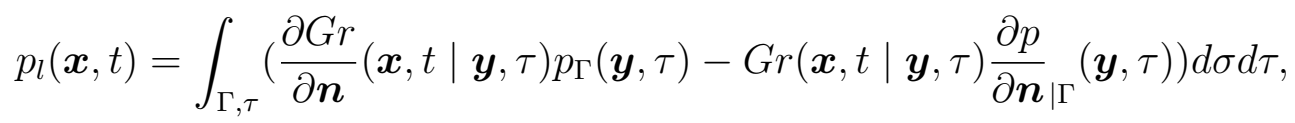

where $\boldsymbol{n}$ is the outward normal to $\Gamma ; G r$ represents Green's function; $\boldsymbol{x}$ correspond to the observation points; the retarded time is considered in the convolution.

Equation (7) can be rewritten in a more compact form via the Calderón potentials (Utyuzhnikov, 2017):

$$
p_{l}(\boldsymbol{x}, t)=P_{D^{+}} \boldsymbol{\xi}_{\Gamma}(\Gamma, t) .
$$

Here, $\boldsymbol{\xi}_{\Gamma}=\left(p_{\Gamma}, \frac{\partial p}{\partial \boldsymbol{n}}{ }_{\Gamma}\right)^{T}$ means the density of the potential.

In application to the wave equation with homogeneous initial conditions, the Calderón potential in domain $\Omega$ is determined by (Utyuzhnikov, 2009a): 


$$
P_{\Omega} \boldsymbol{\xi}_{\partial \Omega}(\boldsymbol{x}, t)=\int_{\partial \Omega, \tau}\left(G r(\boldsymbol{x}, t \mid \boldsymbol{y}, \tau) \xi_{\partial \Omega \mid 2}(\boldsymbol{y}, \tau)-\frac{\partial G r}{\partial \boldsymbol{n}}(\boldsymbol{x}, t \mid \boldsymbol{y}, \tau) \xi_{\partial \Omega \mid 1}(\boldsymbol{y}, \tau)\right) d \sigma d \tau
$$

where $\boldsymbol{x} \in \Omega, \boldsymbol{\xi}_{\partial \Omega}=\left(\xi_{\partial \Omega \mid 1}, \xi_{\partial \Omega \mid 2}\right)^{T}, \boldsymbol{n}$ is the outward normal to $\partial \Omega$.

It is to be noted that for the ASC the initial data are not important. In a more general formulation with nonhomogeneous initial conditions, the definition of the Calderón potential can be found in (Petropavlovsky et al., 2018).

The key property of the Calderón potential is a projection. It immediately follows from the Green's formula (Petropavlovsky et al., 2018; Utyuzhnikov, 2009a). Thanks to the projection property, in the case of continuous surface control

$$
P_{D^{+}} \boldsymbol{\xi}_{\Gamma}(\boldsymbol{x}, t)=p^{-}(\boldsymbol{x}, t), \quad \boldsymbol{x} \in D^{+},
$$

if $\boldsymbol{\xi}_{\Gamma}=\left(p_{\Gamma}, \frac{\partial p}{\partial \boldsymbol{n} \mid \Gamma}\right)^{T}$.

Thus, if the total field from the primary sources $p_{\Gamma}$ is available, then $G_{0}$ generates antinoise and provides complete noise attenuation (see, e.g., (Utyuzhnikov, 2010a)).

The control is local because the surface integrals are approximated in a discrete form via a set of monopoles and dipoles (Utyuzhnikov, 2017):

$$
\left.G_{0}(\Gamma, t) \approx-a^{2} \sum_{i=1}^{N}\left[\frac{\partial p}{\partial \boldsymbol{n}}|\Gamma| \boldsymbol{x}_{i}, t\right) \delta\left(\boldsymbol{x}-\boldsymbol{x}_{i}\right)+p_{\Gamma}\left(\boldsymbol{x}_{i}, t\right) \frac{\partial \delta}{\partial \boldsymbol{n}}\left(\boldsymbol{x}-\boldsymbol{x}_{i}\right)\right] \Delta \sigma_{i}
$$

where $\boldsymbol{x}_{i} \in \Delta \sigma_{i} \quad(i=1, \ldots, N), \boldsymbol{n}$ is the outward normal to $\Gamma, \Delta \sigma_{i} \quad(i=1, \ldots, N)$ are nonintersecting elementary areas $\sigma_{i}$ which cover the entire surface $\Gamma$. 
It is to be noted that from the projection property

$$
P_{D^{+}} \boldsymbol{\xi}_{\Gamma}(\boldsymbol{x}, t)=P_{D^{+}} \boldsymbol{\xi}_{\Gamma}^{-}(\boldsymbol{x}, t),
$$

where $\boldsymbol{\xi}_{\Gamma}^{-}=\left(p_{\Gamma}^{-}\left(\boldsymbol{x}_{i}, t\right), \frac{\partial p^{-}}{\partial \boldsymbol{n}} \mid \Gamma\left(\boldsymbol{x}_{i}, t\right)\right)^{T}$

In turn

$$
P_{D^{+}} \boldsymbol{\xi}_{\Gamma}^{+}(\boldsymbol{x}, t)=0,
$$

where $\left.\boldsymbol{\xi}_{\Gamma}^{+}=\left(p_{\Gamma}^{+}\left(\boldsymbol{x}_{i}, t\right), \frac{\partial p^{+}}{\partial \boldsymbol{n}}|\Gamma| \boldsymbol{x}_{i}, t\right)\right)^{T}$.

Thus, the control looks similar to the case when only noise is present. In the case of pure noise, an anti-noise can be generated locally along the entire perimeter of the protected region. However, this similarity takes place only in the case of continuous surface control. In other words, this presumes the validity of equations (11) and (12) that are actually nonlocal. In the case of a discrete approximation, the projection property is violated up to the error of approximation. This issue is discussed in more detail in the next section.

It is to be noted that if desired sound is present, a field equal to the desired acoustic field $p^{+}$is simultaneously generated by control $G_{0}$ immediately outside the shielded domain (Utyuzhnikov, 2010a). As a result, the primary field is practically unavailable for real-time measurements unless the field is repeated in time. In order to remove the reverse effect of controls, a nonlocal control is introduced in the next section. It is to be noted that the reverse effect occurs only if a desired sound is allowed to be present in the shielded domain. 


$$
\begin{gathered}
p_{e \mid \Gamma}^{-}(\boldsymbol{x}, t)=\int_{\Gamma^{-}, \tau}\left(G r(\boldsymbol{x}, t \mid \boldsymbol{y}, \tau) \frac{\partial p_{c}}{\partial \boldsymbol{n}^{-}}(\boldsymbol{y}, \tau)-\frac{\partial G r}{\partial \boldsymbol{n}^{-}}(\boldsymbol{x}, t \mid \boldsymbol{y}, \tau) p_{c}(\boldsymbol{y}, \tau)\right) d \sigma d \tau, \\
\boldsymbol{x} \in \Gamma, \boldsymbol{y} \in \Gamma^{-}
\end{gathered}
$$

where $\boldsymbol{n}^{-}$is the outward normal to the external surface $\Gamma^{-}$.

In a more general formulation, the field $p_{e}^{-}$can be calculated via the Calderón potentials (Utyuzhnikov, 2017):

$$
p_{e \mid \Gamma}^{-}(\boldsymbol{x}, t)=P_{D_{-}^{+}} \boldsymbol{\xi}_{\Gamma^{-}}\left(\Gamma^{-}, t\right) .
$$

Thanks to the projection property, ideally, i.e. in the case of continuous surface control, $p_{\mid \Gamma}^{-}(\boldsymbol{x}, t)=p_{e \mid \Gamma}^{-}(\boldsymbol{x}, t)$

Thus, the control at the boundary $\Gamma$ is represented by

$$
G_{0}\left(\Gamma, t \mid p_{e \mid \Gamma}^{-}\right)=-a^{2}{\frac{\partial p_{e}}{\partial \boldsymbol{n}}}_{\mid \Gamma}^{-} \delta(\Gamma)-a^{2} \frac{\partial p_{e \mid \Gamma}^{-} \delta(\Gamma)}{\partial \boldsymbol{n}} .
$$


Hence, we can extract the noise field from the total field $p_{c}\left(\Gamma^{-}, t\right)$ on external surface $\Gamma^{-}$ due to the projection property of the surface potentials. As a result, this approach is able to remove the reverse effect of controls on the input data. The obtained field $p_{e}^{-}(\Gamma, t)$ on $\Gamma$ can then be used as the input field for the local control in (6) to achieve noise attenuation. The entire control is non-local in space since its operation at each point of the control surface is based on the total field over the whole surface $\Gamma^{-}$. Besides, it is clear that the control is also nonlocal in time.

It is to be noted that in the general case Green's function is unknown. Therefore, practically control (13) cannot be applicable straightforward.

In order to approximate the integrals in (13), a discrete distribution of sensors and controls is considered in (Utyuzhnikov, 2017). The external surface $\Gamma^{-}$and boundary surface $\Gamma$ are divided into small enough nonintersecting areas $\Delta \sigma_{j}^{-}$and $\Delta \sigma_{i}$, respectively. Presume that $l_{j}^{-}=\sqrt{\Delta \sigma_{j}^{-}} \ll \boldsymbol{r}_{j}=\left|\boldsymbol{y}-\boldsymbol{y}_{j}\right| \quad\left(j=1, \ldots, N^{-}\right), l_{i}=\sqrt{\Delta \sigma_{i}} \ll \boldsymbol{r}_{i}=\left|\boldsymbol{x}-\boldsymbol{x}_{i}\right| \quad(i=1, \ldots, N)$. Next, (13) can be approximated as follows:

$$
p_{e}^{-}\left(\boldsymbol{x}_{i}, t\right) \approx \sum_{j=1}^{N^{-}} \Delta \sigma_{j}^{-} \int_{\tau}\left(\frac{\partial p_{c}}{\partial \boldsymbol{n}^{-}}\left(\boldsymbol{y}_{j}, \tau\right) G r\left(\boldsymbol{x}_{i}, t \mid \boldsymbol{y}_{j}, \tau\right)-p_{c}\left(\boldsymbol{y}_{j}, \tau\right) \frac{\partial G r}{\partial \boldsymbol{n}^{-}}\left(\boldsymbol{x}_{i}, t \mid \boldsymbol{y}_{j}, \tau\right)\right) d \tau
$$

where $\boldsymbol{x}_{i} \in \Delta \sigma_{i} \quad(i=1, \ldots, N), \boldsymbol{y}_{j} \in \Delta \sigma_{j}^{-} \quad\left(j=1, \ldots, N^{-}\right)$.

Then

$$
G_{0}\left(\Gamma, t \mid p_{e \mid \Gamma}^{-}\right) \approx-a^{2} \sum_{i=1}^{N} \Delta \sigma_{i}\left(\frac{\partial p_{e}^{-}}{\partial \boldsymbol{n}}\left(\boldsymbol{x}_{i}, t\right) \delta\left(\boldsymbol{x}-\boldsymbol{x}_{i}\right)+p_{e}^{-}\left(\boldsymbol{x}_{i}, t\right) \frac{\partial \delta}{\partial \boldsymbol{n}}\left(\boldsymbol{x}-\boldsymbol{x}_{i}\right)\right)
$$

where $\boldsymbol{n}$ is the outward normal to $\Gamma$.

Then, the explicit formula for the nonlocal control reads : 


$$
\begin{aligned}
& G_{0}=-a^{2} \sum_{i=1}^{N} \Delta \sigma_{i} * \\
& \sum_{j=1}^{N^{-}} \Delta \sigma_{j}^{-} \int_{t}\left(A_{i j} \frac{\partial p_{c}}{\partial \boldsymbol{n}^{-}}+B_{i j} p_{c}\right)_{\mid \boldsymbol{y}_{j, \tau}} \delta\left(\boldsymbol{x}-\boldsymbol{x}_{i}\right)+\left(C_{i j} \frac{\partial p_{c}}{\partial \boldsymbol{n}^{-}}+D_{i j} p_{c}\right)_{\mid \boldsymbol{y}_{j, \tau}} \frac{\partial \delta}{\partial \boldsymbol{n}}\left(\boldsymbol{x}-\boldsymbol{x}_{i}\right) d \tau .
\end{aligned}
$$

Here, transfer matrices $A, B, C$ and $D$, which have dimensions $\left[N \times N^{-}\right]$, depend only on Green's function and the coordinates of sensors and controls if the geometry of boundary surfaces $\Gamma$ and $\Gamma^{-}$is known. Thus, these matrices can be measured or calculated efficiently in advance with the use of the method of Difference Potentials (Petropavlovsky et al., 2018; Ryaben'kii, 2012).

It is worth noting here that the entire algorithm is based on the projection property of the surface potentials. It presumes the controls and sensors are distributed over whole boundaries $\Gamma$ and $\Gamma^{-}$, respectively. In case only on a selected part of the boundary is covered, the projection property can be either significantly violated or not valid.

\section{Calculation of surface potentials}

The unsteady surface potentials can be efficiently calculated with implementation of the impulse response function that represents an unit impulse applied to a small enough period of time. In the algorithm, the input field on external surface $\Gamma^{-}$is approximated by a set of basis functions $\Phi_{k}(\boldsymbol{y})$ and $\frac{\partial \Phi_{k}}{\partial \boldsymbol{n}^{-}}(\boldsymbol{y}), \boldsymbol{y} \in \Gamma^{-}, k=1, \ldots, N_{b}$, where $N_{b} \ll N^{-}$. Then, an impulse response function can be determined as follows: 


$$
v_{k}\left(\boldsymbol{x}_{i}, t\right)=\left\{\begin{array}{l}
\quad \sum_{j=1}^{N^{-}} \Delta \sigma_{j}^{-}\left(\frac{\partial \Phi_{k}}{\partial \boldsymbol{n}^{-}}\left(\boldsymbol{y}_{j}\right) G r\left(\boldsymbol{x}_{i}, t \mid \boldsymbol{y}_{j}, 0\right)-\Phi_{k}\left(\boldsymbol{y}_{j}\right) \frac{\partial G r}{\partial \boldsymbol{n}^{-}}\left(\boldsymbol{x}_{i}, t \mid \boldsymbol{y}_{j}, 0\right)\right), \\
\quad \text { if } \min _{j} \tau_{i j} \leq t \leq \max _{j} \tau_{i j} \\
0 \text { else, }
\end{array}\right.
$$

where $\boldsymbol{x}_{i} \in \Delta \sigma_{i} \quad(i=1, \ldots, N) ; \boldsymbol{y}_{j} \in \Delta \sigma_{j}^{-} \quad\left(j=1, \ldots, N^{-}\right) ; \boldsymbol{n}^{-}$is the outward normal to $\Gamma^{-}$; $\tau_{i j}=\frac{\left|\boldsymbol{x}_{i}-\boldsymbol{x}_{j}\right|}{a}$ represents a retarded time.

The impulse response functions can be preliminary either calculated or measured. Next, at each time moment $t=t_{p}(p=0,1,2,3 \ldots \ldots)$ density $\boldsymbol{\xi}_{\Gamma^{-}}\left(t_{p}\right)$ can be approximated by solving the following variation problem:

$$
\min _{\alpha_{k}}=\left\|\boldsymbol{\xi}_{\Gamma^{-}}\left(t_{p}\right)-\sum_{k=1}^{N_{b}} \alpha_{k}\left(t_{p}\right) \boldsymbol{\eta}_{k \Gamma^{-}}\right\|_{2}
$$

where $\boldsymbol{\eta}_{k \Gamma^{-}}=\left(\Phi_{k}(\boldsymbol{y}), \frac{\partial \Phi_{k}}{\partial \boldsymbol{n}^{-}}(\boldsymbol{y})\right)^{T}, \boldsymbol{y} \in \Gamma^{-}$.

Then, the surface potentials can be calculated at boundary surface $\Gamma$ :

$$
P_{\Gamma \Gamma^{-}} \boldsymbol{\xi}_{\Gamma^{-}}(t) \approx \sum_{k=1}^{N_{b}} \sum_{l=0}^{p} \alpha_{k}\left(t_{l}\right) v_{k \Gamma}\left(t-t_{l}\right)\left(t_{l+1}-t_{l}\right)
$$

Thus, potential (19) provides for control (15) noise field $p_{e \mid \Gamma}^{-}$to be attenuated.

A more efficient strict way to calculate the unsteady Calderón potentials can be found in (Petropavlovsky et al., 2018). It is based on the partition in time and the existence of lacunas.

In the next section, the results of numerical simulation are presented and discussed. 


\section{RESULTS OF NUMERICAL EXPERIMENTS WITH ASC}

In the setup of numerical simulation, virtual primary sources, sensors and secondary sources are implemented. As domain $D^{+}$to be shielded we consider a cube with the edge length equal to $1 \mathrm{~m}$. Assume the center of domain $D^{+}$coincides with the origin of the Cartesian coordinate system. The boundary surface $\Gamma^{-}$corresponds to the sides of cube $D_{-}^{+}$ which is obtained by the expansion of cube $D^{+}$with the factor of 1.4 in each direction. In this case, the Hausdorff distance between cubes $D^{+}$and $D_{-}^{+}$equals $0.2 \mathrm{~m}$. Assume three virtual secondary sources are evenly distributed along each axis on each side of cube $D^{+}$. In turn, six virtual sensors are implemented along each axis on each side of cube $D^{+}$to measure the total acoustic field.

Three types of virtual primary sources are considered in the numerical simulations: pure tone, mixed sound of three monochromatic sources and broadband signal. In the pure tone test, a noise source with frequency of $50 \mathrm{~Hz}$ is situated outside the shielded domain $\mathrm{D}^{+}$ at point with coordinate $(1.5 \mathrm{~m}, 1.5 \mathrm{~m}, 0 \mathrm{~m})$. In turn, a desired acoustic source with the frequency of $50 \mathrm{~Hz}$ is supposed to be at the center of $\mathrm{D}^{+}$. In addition, we also consider a mixed noise case that is composed of three noise point sources with the frequencies equal to $40 \mathrm{~Hz}, 70 \mathrm{~Hz}$ and $90 \mathrm{~Hz}$, which are situated outside the shielded domain and have coordinates $(1.5 m, 1.5 m, 0 m),(1 m,-1 m, 1 m)$ and $(-1 m, 0.5 m,-1 m)$, respectively. Similarly, the desired mixed sound is composed of three point sources with the frequencies of $40 \mathrm{~Hz}, 70 \mathrm{~Hz}$ and $90 \mathrm{~Hz}$. These point sources are situated inside domain $\mathrm{D}^{+}$and have coordinates $(0.3 m, 0.3 m, 0.3 m),(-0.4 m, 0.1 m, 0.2 m)$ and $(0.2 m,-0.3 m,-0.3 m)$, 
respectively. In the numerical simulations, the sampling rate is chosen as much as $1 \mathrm{kHz}$ to ensure the accuracy of approximation.

In the numerical experiments, an average noise attenuation is measured at $N$ observation points that are evenly distributed in shielded domain $D^{+}$. The level of noise attenuation is determined as follows:

$$
A_{s}=\frac{1}{N} \sum_{k=1}^{N} 20 \log _{10}\left|\frac{p_{k}^{-}}{p_{c \mid k}-p_{k}^{+}}\right| d B,
$$

where $p_{c}$ corresponds to the total field while the control is on.

The noise attenuation achieved with the local and nonlocal controls is discussed in the next section. First, we consider the test case with a monochromatic noise source. Then, the case with a mixed noise field is addressed. It is followed by a sensitivity analysis with respect to the Hausdorff distance $d_{H}$ between surfaces $\Gamma$ and $\Gamma^{-}$as well as the density of sensors $n_{s}$ and controls $n_{c}$. In the final section, we carry out numerical experiments with broadband noise.

\section{A. Pure tone case}

First, consider the conventional ASC algorithm based on the local control for the case of pure-tone primary sources. Presume that the total primary field is a priori known. In case the desired sound is not present, Figure 2(a) shows the $50 \mathrm{~Hz}$ noise field with and without shielding inside domain $D^{+}$for time interval $\left[\begin{array}{llll}0 & s, & 1\end{array}\right]$. The average noise attenuation of about $12 d B$ is achieved over time. Here and further, $N=100$. Thus, the noise is effectively attenuated inside the shielded domain. 
In turn, if the desired sound is present, the noise attenuation deteriorates even though the primary field is available at the boundary surface $\Gamma$. As can be seen from Figure 2(b), the noise field with ASC is only slightly attenuated. The corresponding average noise attenuation drops to $2.5 d B$, which is essentially worse than that in the previous case. The reason for this can be interpreted as follows. As mentioned in the previous section, the local control is determined by the total acoustic field at the perimeter $\Gamma$. The error in the approximation of surface potentials in Eq. (11) leads to some violation of the projection property (12). This error can be treated as an extra noise. Thus, the noise attenuation is deteriorated. 


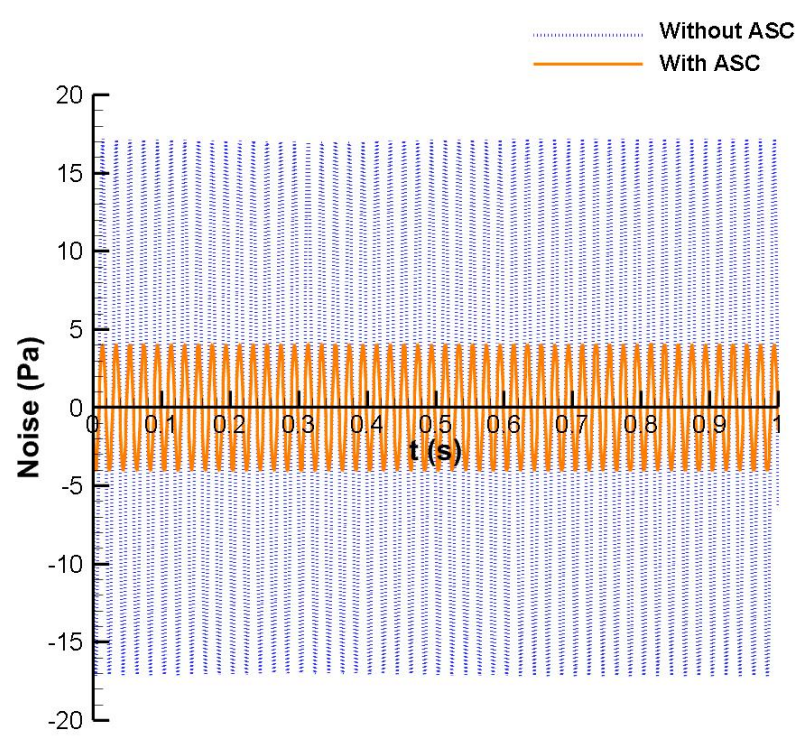

(a)

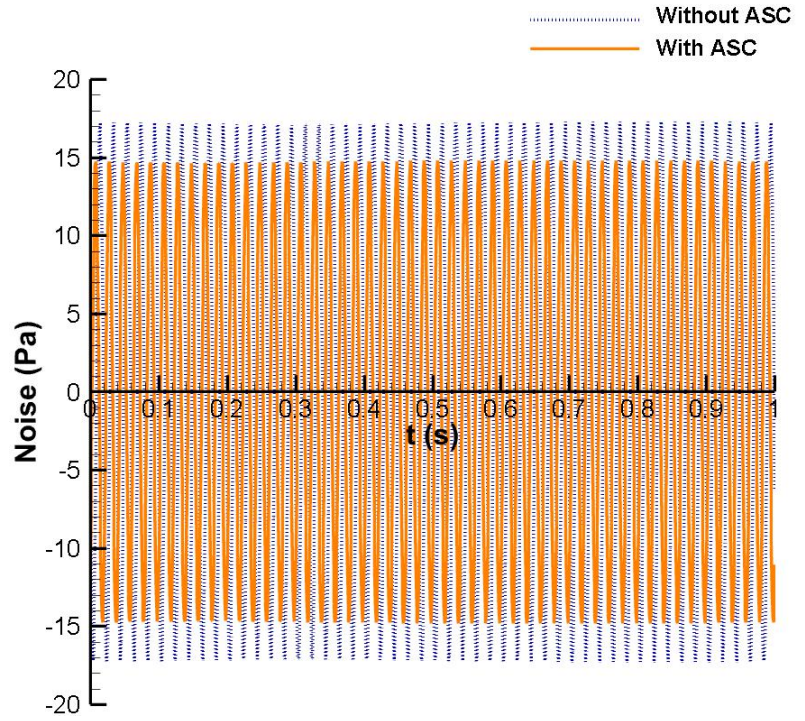

(b)

FIG. 2. Application of local control to a pure tone field with input primary field: (a) Noise signal (without desired sound), (b) Noise signal (with desired sound).

As mentioned above, practically the primary field is not available at the perimeter $\Gamma$ if a desired sound is present due to the reverse effect of controls. Therefore, next a more realistic case that takes into account the reverse effect of controls on the input data is considered. Figure 3(a) shows that the noise is rather reinforced than attenuated with the input data based on the total field in the case of the local control. The primary field is the same as that in the previous example. As can be seen, the noise level growths with time under the effect of the control. This phenomenon can be explained as follows.

Control (6) doubles internally generated sound outside the shielded domain in the case of continuous control (see, e.g., (Utyuzhnikov, 2009a)). Since control (6) must be approximated by discrete control (10), projection (12) is only valid with some error. This error should effectively lead to generation of extra imaginary noise sources caused by the discrete control 
that follows from the Huygens principle. The field generated by the imaginary sources is also reinforced outside the shielded domain as an internally generated sound. This effect is repeated and reinforced with time.

Next, consider the application of nonlocal control to a pure tone field. In contrast to the local control, the input data are measured on $\Gamma^{-}$. In case the desired field is not present, Figure 4 demonstrates the sound pressure achieved at $0.5 \mathrm{~s}$. As can be seen, the sound pressure is efficiently attenuated inside the cubic shielded domain $D^{+}$. Moreover, the amplitude of sound wave achieved with the nonlocal control for time interval $[0 s, 1 s]$ is similar to that obtained with the local control considered above (see Figures 2(a) and Table I).

TABLE I. Application of nonlocal control to a pure tone field with input total field.

The case without desired sound

Amplitude, $\mathrm{Pa}$

Attenuation,

Amplitude, $P a$

Attenuation,

The case with desired sound

\begin{tabular}{|c|c|c|c|c|c|c|}
\hline & \multicolumn{2}{|c|}{ Amplitude, $P a$} & \multirow{2}{*}{$\begin{array}{c}\text { Attenuation, } \\
\quad d B\end{array}$} & \multicolumn{2}{|c|}{ Amplitude, $P a$} & \multirow{2}{*}{$\begin{array}{l}\text { Attenuation } \\
\qquad d B\end{array}$} \\
\hline & (without ASC) & (with ASC) & & (without ASC) & (with ASC) & \\
\hline Noise & 17.10 & 4.60 & 11.40 & 17 & 4.75 & 11.10 \\
\hline
\end{tabular}

In turn, as can be seen in Table I, a good level of noise attenuation is still achieved by the nonlocal control even if the desired sound is present. The corresponding average noise attenuation of $11 \mathrm{~dB}$ basically retains the same as that without the existence of the desired sound source. The contribution of the desired field and extra noise caused by a discrete 


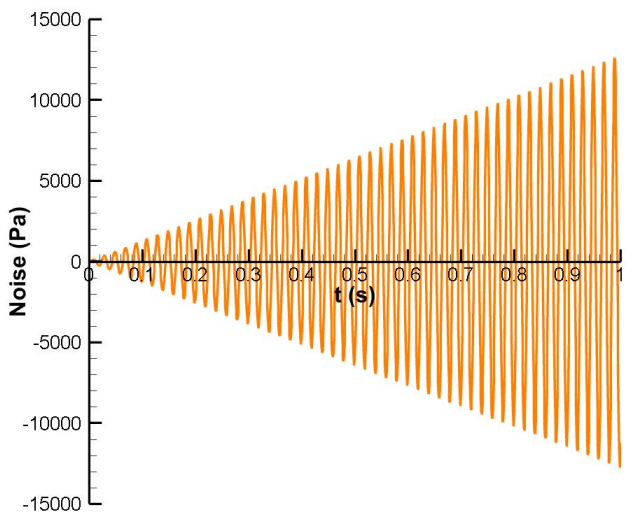

(a)

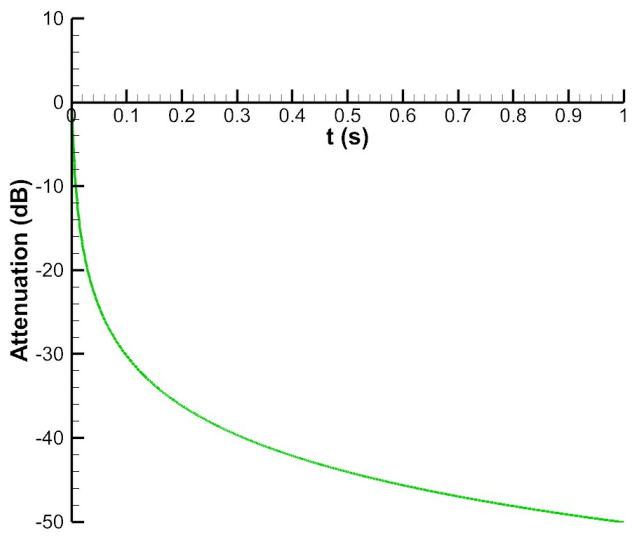

(b)

FIG. 3. Reverse effect of local control with input total field. Pure tone case: (a) Noise level, (b) Level of noise attenuation.

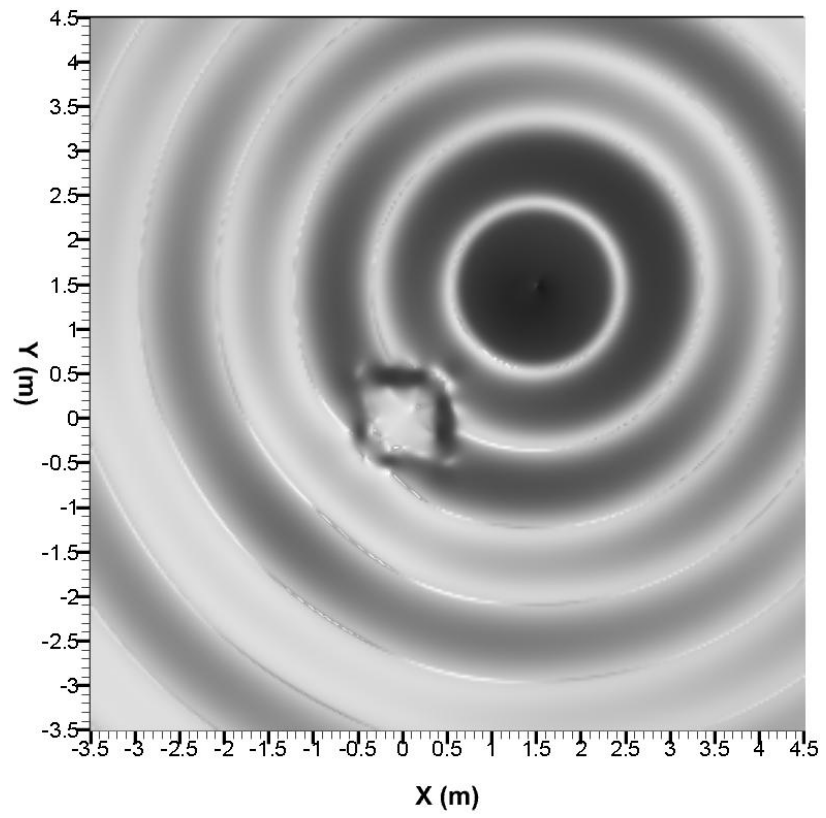

FIG. 4. Sound pressure at $0.5 \mathrm{~s}$. Pure tone case without desired sound. 
It is to be noted that with the nonlocal control the level of noise attenuation is determined by the frequency of noise source and almost does not depend on the frequency of desired sound. This is confirmed by the result that the level of noise attenuation with and without desired sound is practically the same.

\section{B. Mixed sound case}

Next, consider the attenuation of noise composed of three noise point sources with frequencies of $40 \mathrm{~Hz}, 70 \mathrm{~Hz}$ and $90 \mathrm{~Hz}$. In turn, there are three desired sound sources with the same frequencies if they are supposed to be present.

First, the case with local control is studied without the presence of desired sound. Assume that the primary field is always available at perimeter $\Gamma$ over time. Then, as can be seen in Table II, the noise can be significantly attenuated. The level of noise attenuation varies between $7 d B$ and $13 d B$ at different time $(\mathrm{t}=0.015 s, 0.02 s, 0.025 s, 0.03 s, 0.035 s)$. In contrast to the attenuation of pure tone noise, the level of noise attenuation for the mixed case essentially depends on time. This happens because the level of noise attenuation depends on the frequency. It is clear that the higher frequency the lower level of noise attenuation occurs (Epain and Friot, 2007; Zhou and Utyuzhnikov, 2020).

In case the local control is used along with the presence of desired sound, the noise is reinforced similar to the monochromatic case. As shown in Table II, the level of noise attenuation falls to $-6 d B$ at $0.03 s$ due to the additional noise caused by the discrete control. Over time, the negative level of noise attenuation varies between $-6 d B$ and $4 d B$. 
If the primary field is not a priori available on surface $\Gamma$, the control affects the input data measured at $\Gamma$. Similar to the single tone case, this effect continuously growths over time as can be seen in Figure 5(a). Moreover, as Figure 5(b) demonstrates, the noise attenuation becomes practically impossible with the local control.

TABLE II. Application of local control to mixed sound case with input primary field.

\begin{tabular}{|c|c|c|c|c|c|c|}
\hline \multirow{3}{*}{$\mathrm{t}, \mathrm{s}$} & \multicolumn{3}{|c|}{ The case without desired sound } & \multicolumn{3}{|c|}{ The case with desired sound } \\
\hline & \multicolumn{2}{|c|}{ Noise, $P a$} & \multirow{2}{*}{$\begin{array}{l}\text { Attenuation, } \\
\qquad d B\end{array}$} & \multicolumn{2}{|c|}{ Noise, $P a$} & \multirow{2}{*}{$\begin{array}{c}\text { Attenuation, } \\
\qquad d B\end{array}$} \\
\hline & (without ASC) & (with ASC) & & (without ASC) & (with ASC) & \\
\hline 0.015 & -33.45 & 8.48 & 12 & -33.45 & -35.38 & -0.5 \\
\hline 0.02 & 58.63 & -20.41 & 9 & 58.6 & -38.46 & 4 \\
\hline 0.025 & 9.96 & 4.27 & 7 & 9.96 & 12.07 & -2 \\
\hline 0.03 & 37.06 & -11.47 & 10 & 37.06 & -75.55 & -6 \\
\hline 0.035 & -39.86 & 9.29 & 13 & -39.86 & 34.11 & 1 \\
\hline
\end{tabular}

Afterwards, consider the nonlocal control applied to the mixed field. First, Table III demonstrates that the level of noise signal with the active control is significantly less than the original noise signal in case there are no interior sources. The peak of the noise attenuation reaches about $13 \mathrm{~dB}$ at $0.035 \mathrm{~s}$. In turn, if the preservation of the interior desired sound in domain $D^{+}$is required, the noise can significantly be reduced with the nonlocal control. Moreover, although the noise attenuation varies over time, the level of noise attenuation 


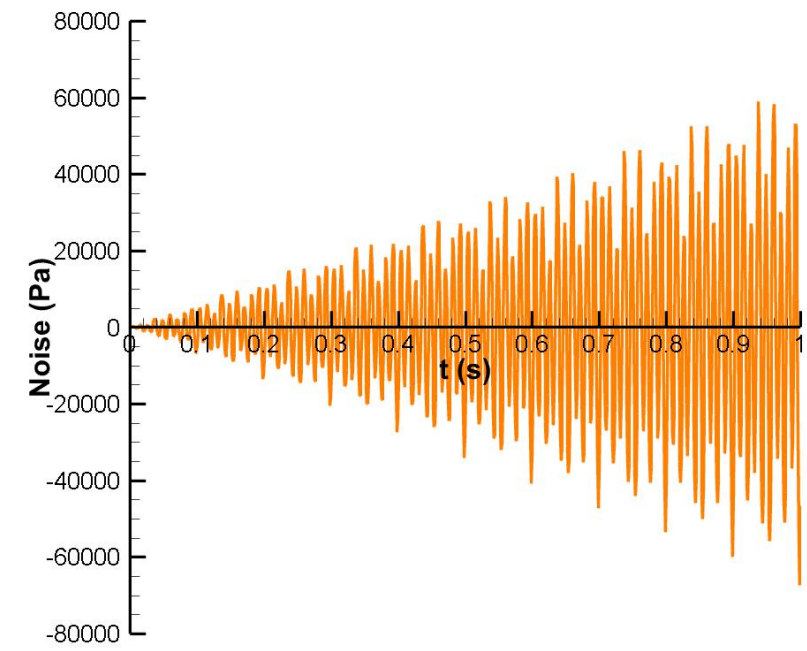

(a)

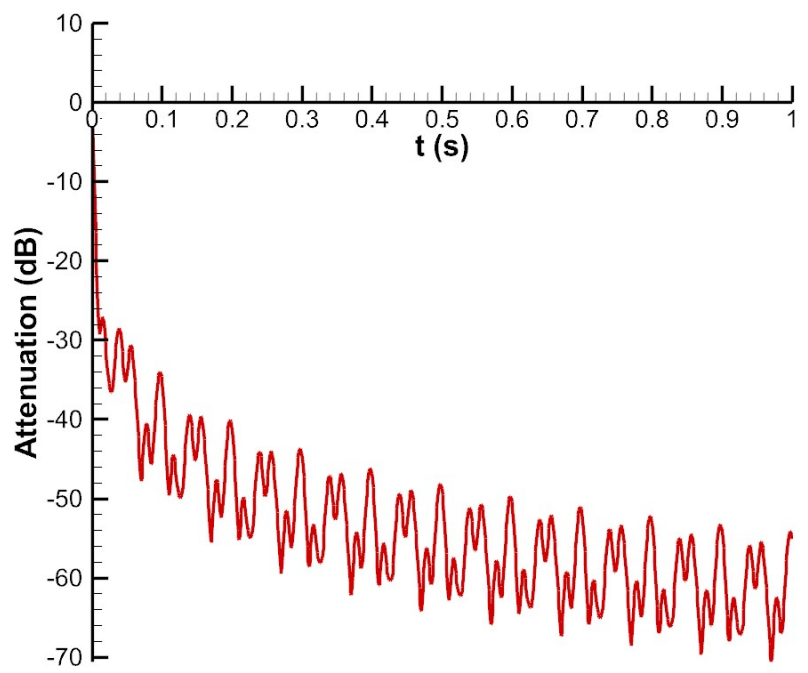

(b)

FIG. 5. Reverse effect of local controls on noise attenuation. Mixed sound: (a) Noise, (b) Level of noise attenuation. 
TABLE III. Application of nonlocal control to mixed sound case with input primary field.

\begin{tabular}{|c|c|c|c|c|c|c|}
\hline \multirow{3}{*}{$\mathrm{t}, s$} & \multicolumn{3}{|c|}{ The case without desired sound } & \multicolumn{3}{|c|}{ The case with desired sound } \\
\hline & \multicolumn{2}{|c|}{ Noise, $P a$} & \multirow{2}{*}{$\begin{array}{c}\text { Attenuation, } \\
d B\end{array}$} & \multicolumn{2}{|c|}{ Noise, $P a$} & \multirow{2}{*}{$\begin{array}{l}\text { Attenuation, } \\
\qquad d B\end{array}$} \\
\hline & (without ASC) & (with ASC) & & (without ASC) & (with ASC) & \\
\hline 0.015 & -33.45 & 9.91 & 11 & -33.45 & 9.56 & 11 \\
\hline 0.02 & 58.63 & -22.66 & 8 & 58.6 & -21.52 & 9 \\
\hline 0.025 & 9.96 & -4.70 & 7 & 9.96 & -4.73 & 7 \\
\hline 0.03 & 37.06 & -12.73 & 9 & 37.06 & -12.10 & 10 \\
\hline 0.035 & -39.86 & 9.43 & 13 & -39.86 & 9.35 & 13 \\
\hline
\end{tabular}




\section{Sensitivity analysis}

As mentioned in the previous sections, the level of noise attenuation significantly depends on the errors in the discrete approximation of the surface potentials. Practically, both the sensors and controls (loudspeakers) cannot be implemented continuously. Therefore, it is important to discuss how the densities of sensors $n_{s}$ and controls $n_{c}$ affect the ASC performance. Besides, the Hausdorff distance $d_{H}$ between the external surface $\Gamma^{-}$and boundary surface $\Gamma$ has an effect on the noise attenuation. This section focuses on the sensitivity analysis with respect to these factors via some numerical experiments. In the tests, the pure tone and mixed primary fields are considered.

First, we study how the density of sensors affects the noise attenuation. Suppose that the number of controls along each axis $N_{c}$ is equal to 3 . In other words, 9 controls on each side and 54 controls in total are distributed on surface $\Gamma$. Several sets of sensors with $N_{s}=3,4,5,6$ are implemented on external surface $\Gamma^{-}$to detect the input field.

TABLE IV. Effect of the number of sensors $N_{s}$ on attenuation of pure tone sound.

\begin{tabular}{lcccc}
\hline \hline & \multicolumn{3}{c}{$N_{s}$} \\
\cline { 2 - 5 } & 3 & 4 & 5 & 6 \\
\hline Noise attenuation, $d B$ & -9.83 & 4.34 & 7.53 & 11.10 \\
\hline \hline
\end{tabular}

As can be seen in Table IV and Figures 6, a poor noise attenuation is achieved with pure tone and mixed primary field in the case with $N_{s}=3, N_{c}=3$. The noise attenuation even 
falls to $-9.83 d B$ with the pure tone primary field in time interval $[0 s, 1 s]$. Besides, the level of noise attenuation occurs mostly in a negative area with the mixed primary field. Moreover, a satisfactory noise attenuation is not achieved when the number of sensors along each axis $N_{s}$ is equal to 4 and 5 . In turn, about $11 d B$ reduction in the noise level is achieved if $N_{s}$ increases to 6 . It is clear that the error in the discrete presentation of sensors (13) is reduced as the higher density of sensors is implemented. It turns out that the number of sensors along each axis $N_{s}$ is required to be twice more than the number of controls along each axis $N_{c}$ for the most efficient noise attenuation.

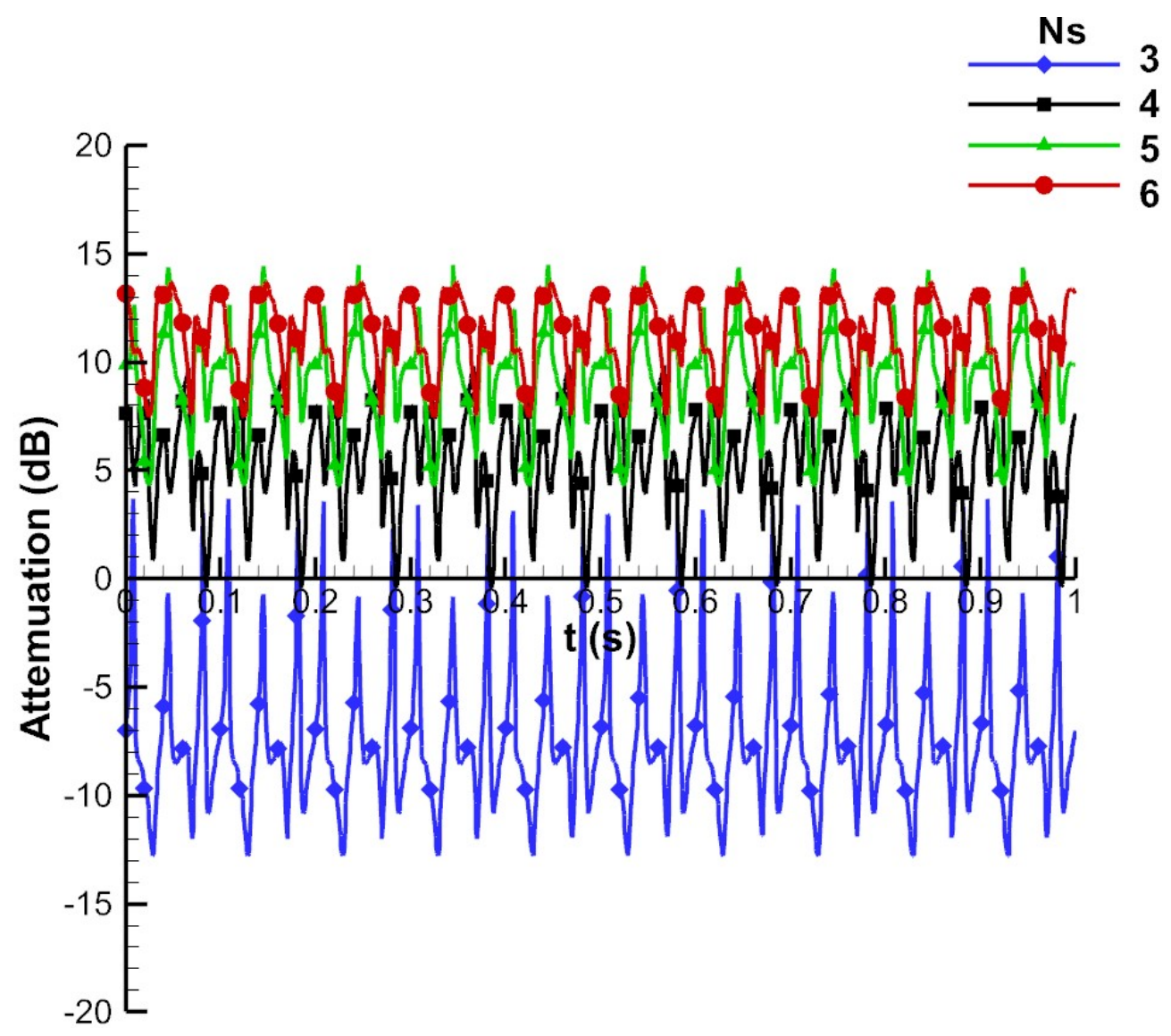

FIG. 6. Effect of the number of sensors $N_{s}$ on attenuation of mixed sound. 
Next, consider the sensitivity analysis with respect to the density of controls. Several sets of controls $\left(N_{c}=2,3,4,5\right)$ are uniformly distributed on the boundary surface $\Gamma$ while $N_{s}$ is equal to 10. First, presume that the total primary field is known for ASC. Table V and VI show the effect of the number of controls on the attenuation of pure tone and mixed noise, respectively.

TABLE V. Effect of the number of controls $N_{c}$ on attenuation of pure tone sound.

\begin{tabular}{|c|c|c|c|c|}
\hline \multirow[b]{2}{*}{$N_{c}$} & \multicolumn{4}{|c|}{ Noise attenuation, $d B$} \\
\hline & $\begin{array}{c}\text { local control } \\
\text { (without desired sound) }\end{array}$ & $\begin{array}{c}\text { local control } \\
\text { (with desired sound) }\end{array}$ & $\begin{array}{c}\text { Nonlocal control } \\
\text { (without desired sound) }\end{array}$ & $\begin{array}{l}\text { Nonlocal control } \\
\text { (with desired sound) }\end{array}$ \\
\hline 2 & 9.56 & 0.69 & 8.98 & 9.02 \\
\hline 3 & 11.70 & 2.63 & 11.40 & 11.10 \\
\hline 4 & 14.46 & 5.39 & 14.50 & 14.63 \\
\hline 5 & 17.28 & 8.22 & 17.74 & 17.87 \\
\hline
\end{tabular}

As noted above, if a desired sound is present the noise attenuation with the local control is much worse than that achieved without the presence of desired field. As shown in Table $\mathrm{V}$, a good enough noise attenuation of $9.56 \mathrm{~dB}$ is achieved with the local control without the presence of desired sources if $N_{c}$ equals 2. However, if the desired sound source is present, the noise attenuation drops to $0.69 d B$. The deterioration of noise attenuation is about 9 $d B$. The same level of drop retains in case $N_{c}$ increases to 3,4 and 5 . In turn, the nonlocal control allows us to keep a satisfactory level of noise attenuation despite the presence of the desired sound. Even in case $N_{c}$ equals 2, the noise attenuation obtained with and without preservation of desired field corresponds to $9.02 d B$ and $8.98 d B$, respectively. The level of 
noise attenuation remains low sensitive to the presence of desired sound with the other sets of controls: $N_{c}=3,4,5$.

In the mixed field case, the noise attenuation caused by the local control varies between $13 d B$ and $18 d B$ without the presence of desired sound if $N_{c}$ equals 5 (see Table VI). In case we consider the preservation of desired sound, the deterioration in the noise attenuation growths with the increase of $N_{c}$. The peak of noise attenuation even falls to a negative value, about - $12 d B$, if $N_{c}$ reduces to 2 . This means that the mixed noise is enhanced rather than reduced if the local control is applied. In addition, the level of noise attenuation fluctuates more significantly with the lower density of controls. In turn, in case the nonlocal control is applied to the mixed field, as shown in Table VI, a good enough level of noise attenuation, $13-19 d B$, is achieved. It retains the same regardless the presence of desired sources if $N_{c}$ is equal to 5 . Even if $N_{c}$ decreases to 2 , the noise attenuation is quite reasonable. The reason for this is similar to the pure tone case discussed above.

TABLE VI. Effect of the number of controls $N_{c}$ on attenuation of mixed sound.

\begin{tabular}{|c|c|c|c|c|}
\hline \multirow[b]{2}{*}{$N_{c}$} & \multicolumn{4}{|c|}{ Interval of noise attenuation, $d B$} \\
\hline & $\begin{array}{c}\text { local control } \\
\text { (without desired sound) }\end{array}$ & $\begin{array}{c}\text { local control } \\
\text { (with desired sound) }\end{array}$ & $\begin{array}{c}\text { Nonlocal control } \\
\text { (without desired sound) }\end{array}$ & $\begin{array}{l}\text { Nonlocal control } \\
\text { (with desired sound) }\end{array}$ \\
\hline 2 & $5-11$ & $-12--1$ & $5-11$ & $5-11$ \\
\hline 3 & $7-13$ & $-7-4$ & $7-13$ & $7-13$ \\
\hline 4 & $10-16$ & $-3-6$ & $9-16$ & $10-16$ \\
\hline 5 & $13-18$ & $0-9$ & $13-19$ & $13-19$ \\
\hline
\end{tabular}


Finally, we consider how the ratio of Hausdorff distance $d_{H}$ between surfaces $\Gamma$ and $\Gamma^{-}$ to wave length $\lambda$ affects the noise attenuation achieved with the use of nonlocal control. Suppose that the number of controls $N_{c}$ and sensors $N_{s}$ is equal to 3 and 6 , for the sake of comparison. First, Table VII presents the noise attenuation for the pure tone field with $\lambda=10 \mathrm{~m}$ achieved for different Hausdorff distances $\left(d_{H}=0.1 \mathrm{~m}, 0.2 \mathrm{~m}, \ldots, 0.9 \mathrm{~m}\right) . \mathrm{A}$ significant noise attenuation is obtained for the pure tone field if the ratio $d_{H} / \lambda$ is above 0.02 . The noise attenuation increases with the increase of ratio $d_{H} / \lambda$ until it reaches a maximal value of $13.21 d B$ with $d_{H} / \lambda=0.08$. Afterwards, the noise attenuation decreases slightly to $13.18 d B$ if the ratio $d_{H} / \lambda$ increases to 0.09 . Thus, optimal ratio $d_{H} / \lambda$ is equal to 0.08 in this case. In turn, in case $d_{H} / \lambda$ is equal to 0.01 , a negative noise attenuation of about -5 $d B$ is obtained in time interval $[0 s, 1 s]$. The deterioration in the noise attenuation occurs because the operation of nonlocal control becomes rather local in case the external surface $\Gamma^{-}$is too close to the boundary surface $\Gamma$. As demonstrated in (Zhou and Utyuzhnikov, 2020), a control point source operates locally in its some vicinity.

TABLE VII. Effect of the ratio between Hausdorff distance $d_{H}$ and wave length $\lambda$ on attenuation of pure tone sound.

\begin{tabular}{lcccccccccc}
\hline \hline & \multicolumn{1}{c}{$d_{H} / \lambda$} \\
\cline { 2 - 10 } & 0.01 & 0.02 & 0.03 & 0.04 & 0.05 & 0.06 & 0.07 & 0.08 & 0.09 \\
\hline Noise attenuation, $d B$ & -4.91 & 11.10 & 11.88 & 12.07 & 12.22 & 12.43 & 12.83 & 13.21 & 13.18 \\
\hline \hline
\end{tabular}


Next, Figure 7 demonstrates the noise attenuation for the mixed primary fields achieved for different Hausdorff distances $\left(d_{H}=0.1 \mathrm{~m}, 0.15 \mathrm{~m}, 0.2 \mathrm{~m}, 0.25 \mathrm{~m}\right)$. The minimal wave length $\lambda_{\min }$ is used as the reference length. As can be seen, the mixed noise is effectively attenuated if the ratio $d_{H} / \lambda_{\min }$ equals 0.06 . There is a slight increase of noise attenuation if $d_{H} / \lambda_{\min }$ decreases to 0.05 . The level of noise attenuation retains if $d_{H} / \lambda_{\min }$ is equal to 0.04. In turn, the noise attenuation achieved with $d_{H} / \lambda_{\min }=0.03$ reaches its minimum of $-8 d B$. Thus, the optimal Hausdorff distance $d_{H}$ between surfaces $\Gamma$ and $\Gamma^{-}$is about $5 \%$ of the minimal wave length to be attenuated.

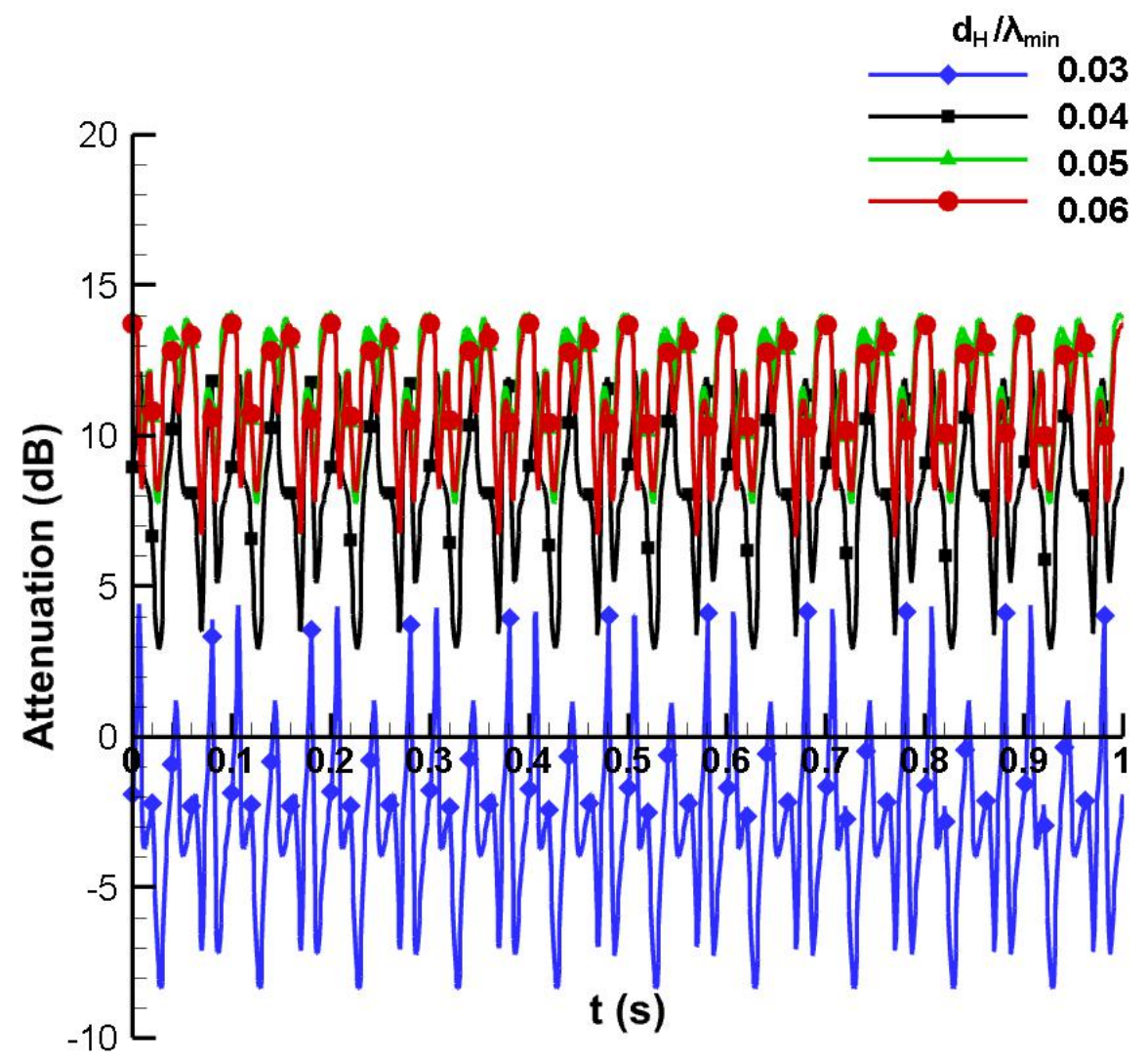

FIG. 7. Effect of the ratio between Hausdorff distance $d_{H}$ and $\lambda_{\min }$ on attenuation of mixed sound. In the final section, the technique is extended to the case of broadband noise. 


\section{BROADBAND SOUND}

Consider now the case of broadband noise in the range below $100 \mathrm{~Hz}$. The coordinates of noise and desired sources, parameters of shielded domain, density of controls and sensors, Hausdorff distance are the same as those in the pure tone case. The considered desired sound wave is monochromatic with the frequency of $50 \mathrm{~Hz}$. Figure 8 represents the sound pressure obtained at $0.5 s$ with the preservation of desired sound. As can be seen, the controls are individually distinguishable at perimeter $\Gamma$. With the application of controls, the desired sound is preserved inside domain $D^{+}$while the noise is shielded. It is to be noted here that the results presented for very low frequencies down to $0 \mathrm{~Hz}$ are rather formal since such frequencies are hardly achievable in practice.

If the desired sound is not present, the broadband noise with and without shielding inside domain $D^{+}$for time interval $[0 s, 1 s]$ is shown in Figure 9 (a). As can be seen, the broadband noise can also be effectively attenuated. In turn, despite the presence of the desired sound, as can be seen in Figure 9(b), the noise attenuation obtained with the nonlocal control is basically the same as that without the desired sound.

The corresponding frequency spectrums with and without preservation of desired sound are shown in Figures 9(c) and 9(d), respectively. At low frequencies, especially below 40 $H z$, a significant noise attenuation is reached. It decreases as the frequency increases. It should be noted that the noise with ASC is attenuated slightly or even enhanced when the frequency exceeds $90 \mathrm{~Hz}$. Next, Figures $9(\mathrm{f})$ and $9(\mathrm{e})$ present the spectrum of noise attenuation obtained with and without presence of desired sound, respectively. Above $3 d B$, 

nonlocal control.

the noise attenuation can be reached with frequencies below $40 \mathrm{~Hz}$. In turn, a negative level of noise attenuation, about $-0.05 \mathrm{~dB}$, is obtained at $100 \mathrm{~Hz}$. As can be seen, the presence of desired sound weakly affects the level of noise attenuation. This is a key property of the

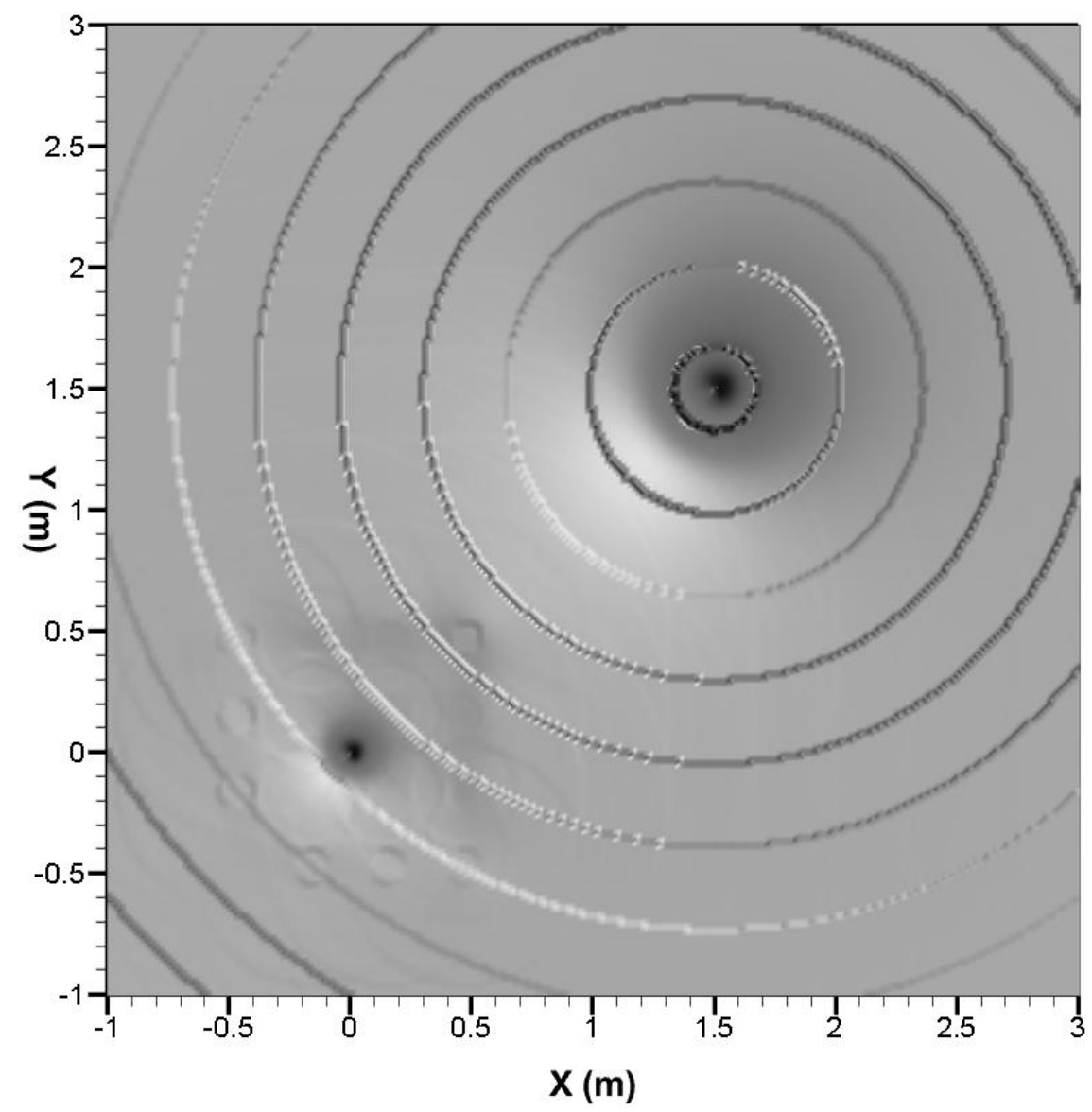

FIG. 8. Sound pressure at $0.5 \mathrm{~s}$. Broadband sound case with desired sound.

Next, consider the sensitivity analysis with respect to the ratio between the Hausdorff distance $d_{H}$ and minimal wave length $\lambda_{\min }$ to be attenuated. Since the set up of the broadband case is the same as that in pure tone case, the reference Hausdorff distance $d_{H}$ and its corresponding ratio $d_{H} / \lambda_{\min }$ are equal to $0.2 \mathrm{~m}$ and 0.06 . Several tests with different ratios 
423

$d_{H} / \lambda_{\min }\left(d_{H} / \lambda_{\min }=0.05,0.06,0.07,0.08\right)$ are considered while the densities of controls $N_{c}$ and sensors $N_{s}$ remain the same as those in the monochromatic case $\left(N_{c}=3, N_{s}=6\right)$. Figure 10 shows the frequency spectrum of noise attenuation reached with several sets of ratio $d_{H} / \lambda_{\min }$ in case the desired sound is present. First, a negative value of noise attenuation is obtained with $d_{H} / \lambda_{\min }=0.07$ and 0.08 if frequency is above 65 and $55 \mathrm{~Hz}$, respectively. In turn, a better noise attenuation is achieved with ratio $d_{H} / \lambda_{\min }$ of 0.06 than that obtained with ratio $d_{H} / \lambda_{\min }$ of 0.07 and 0.08 in the frequency spectrum. In case ratio $d_{H} / \lambda_{\min }$ decreases to 0.05 , a negative peak of noise attenuation, about $-6 \mathrm{~dB}$, is observed at $50 \mathrm{~Hz}$. This phenomenon occurs because the contribution of the desired sound is not completely removed from the total field on the external surface $\Gamma^{-}$. Moreover, the operation of controls becomes local since the external surface $\Gamma^{-}$is too close to the boundary surface $\Gamma$. Thus, the optimal ratio $d_{H} / \lambda_{\min }$ equals 0.06 in this case.

Afterwards, consider the sensitivity analysis with respect to the density of sensors $N_{s}$. Suppose that $d_{H} / \lambda_{\min }$ and $N_{c}$ are equal to 0.06 and 3 , respectively. Figure 11 demonstrates the frequency spectrum of noise attenuation obtained with several sets of $N_{s}\left(N_{s}=4,5,6,7\right)$ if the desired sound is present. In the cases of $N_{s}=4,5$ the density of sensors is not large enough such that the deterioration of noise attenuation occurs between $-2 d B$ and $0.8 d B$ if the frequency exceeds $50 \mathrm{~Hz}$. If $N_{s}$ increases to 6 , even though a good enough noise attenuation is reached for frequencies below $50 \mathrm{~Hz}$, the noise attenuation deteriorates to a negative value for frequencies above $95 \mathrm{~Hz}$. In turn, a better noise attenuation, above $1 \mathrm{~dB}$, is obtained with $N_{s}=7$ for higher frequencies in comparison with $N_{s}=6$. 


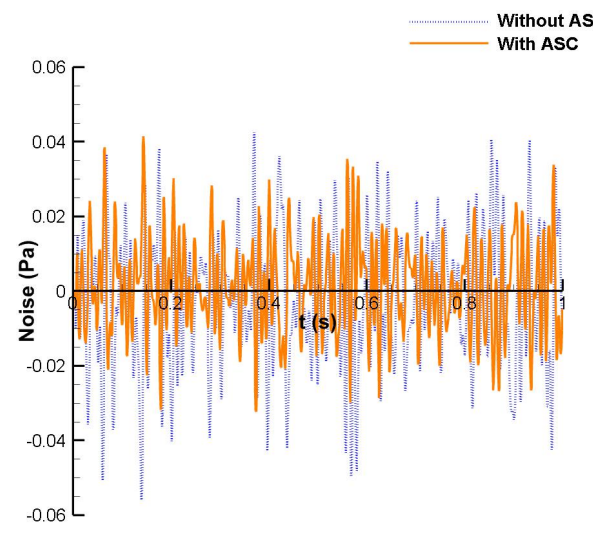

(a)

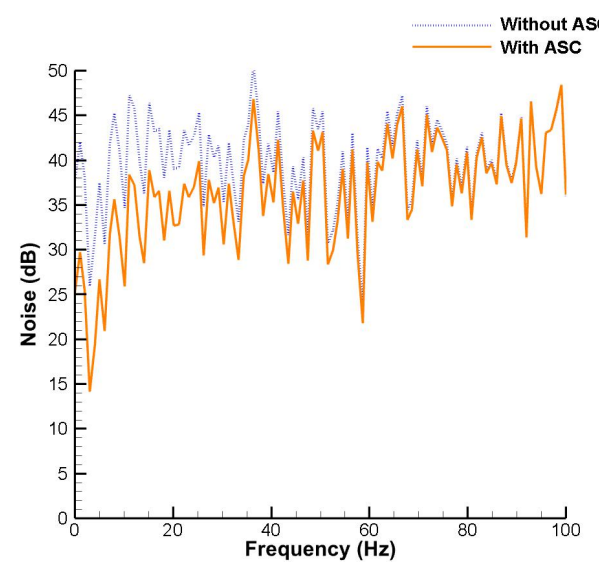

(c)

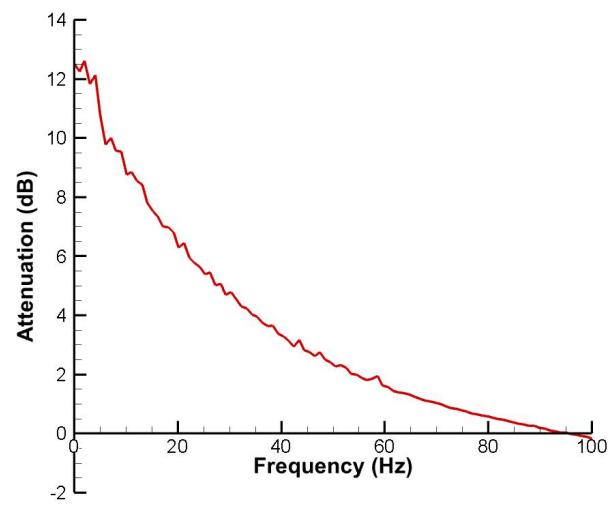

(e)

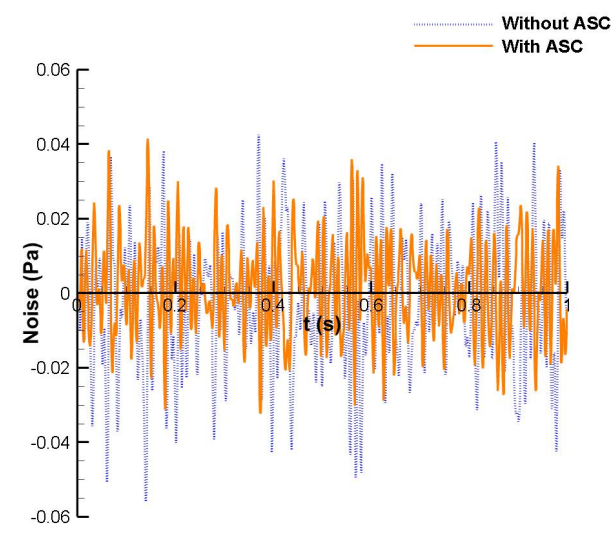

(b)

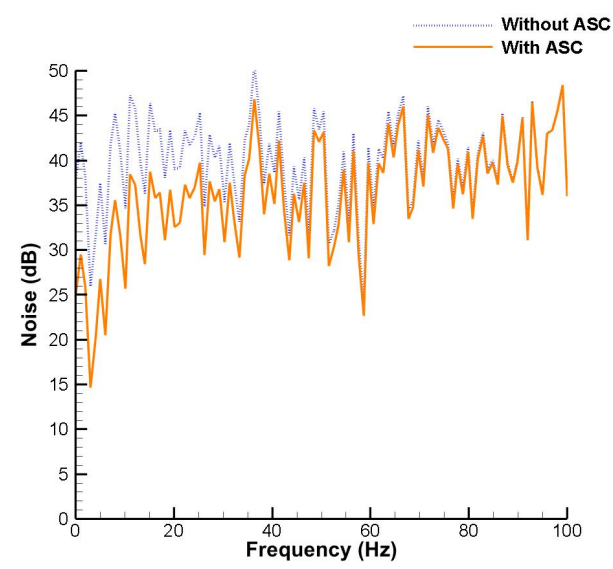

(d)

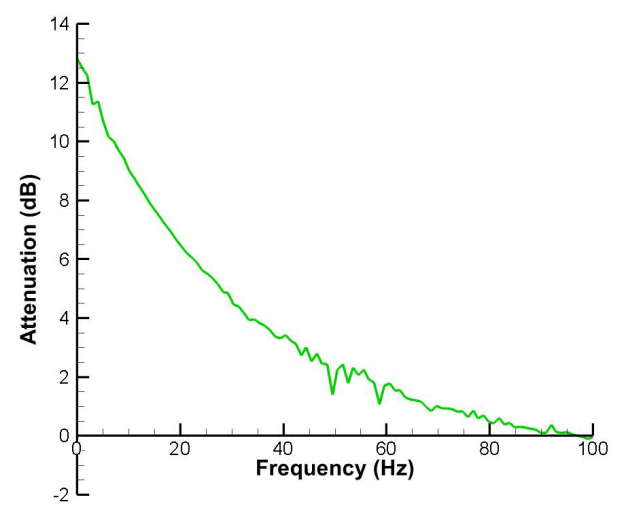

(f)

FIG. 9. Application of nonlocal control on broadband sound case with input primary field: (a)

Noise signal (without desired sound), (b) Noise signal (with desired sound); (c) Frequency spectrum (without desired sound); (d) Frequency spectrum (without desired sound); (e) Noise attenuation (without desired sound); (f) Noise attenuation (with desired sound). 


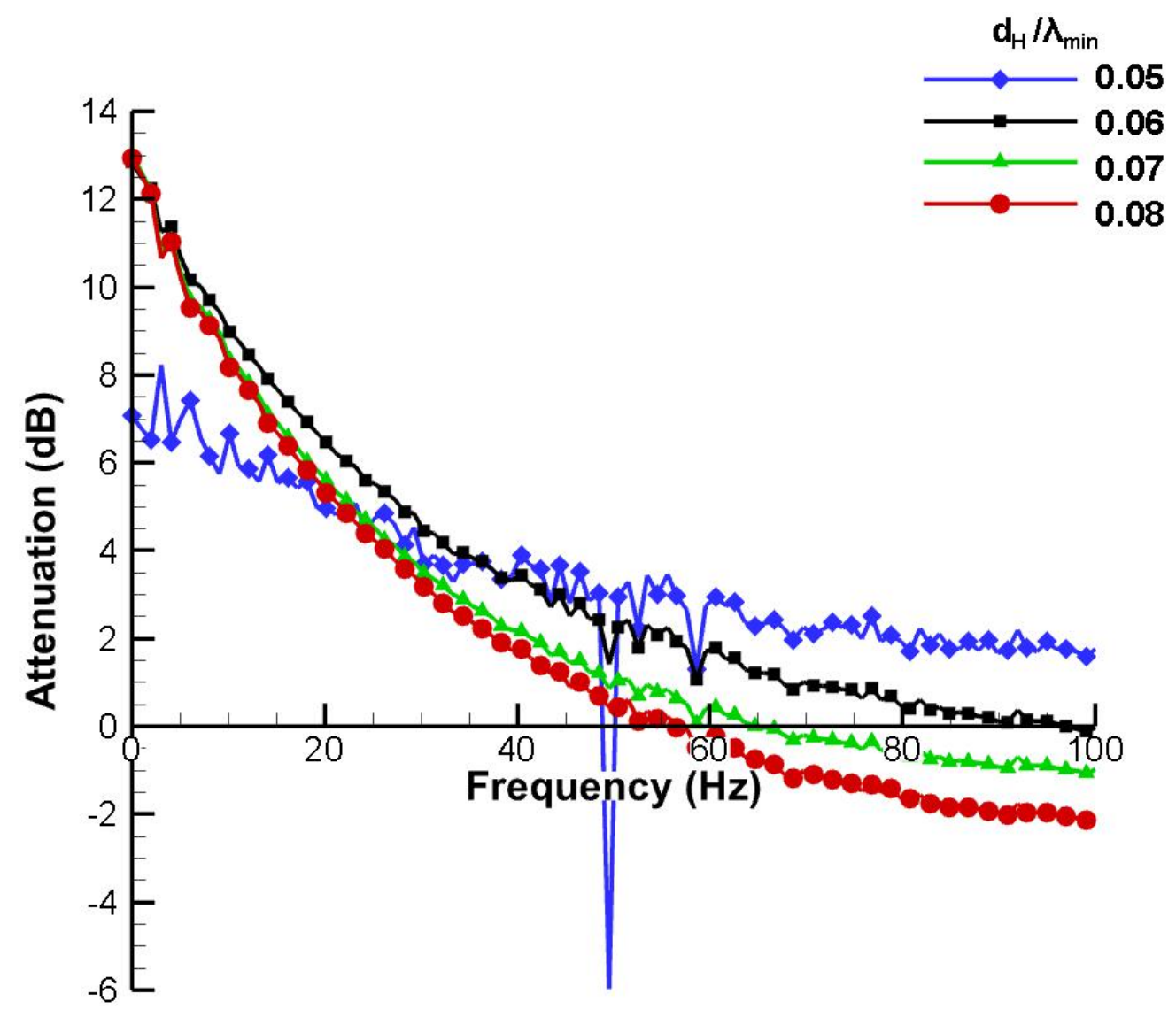

FIG. 10. Effect of the ratio between Hausdorff distance $d_{H}$ and $\lambda_{\min }$ on attenuation of broadband noise.

Finally, consider how the density of controls $N_{c}$ affects the noise attenuation. Assume that $d_{H} / \lambda_{\min }$ and $N_{s}$ are equal to 0.06 and 6 , respectively. Figure 12 presents the frequency spectrum of noise attenuation reached with several sets of the density of controls $N_{c}$ ( $N_{c}=$ $2,3,4,5)$ in case the desired sound is present. A satisfactory noise attenuation is reached in the frequency range up to $100 \mathrm{~Hz}$ if $N_{c}$ equals 5. Significant noise attenuation is reached at very low frequencies. In turn, the noise attenuation deteriorates to around $0 d B$ at higher frequencies. Finally, a negative level of noise attenuation is obtained with $N_{c}=2$ if the frequency is above $80 \mathrm{~Hz}$. 


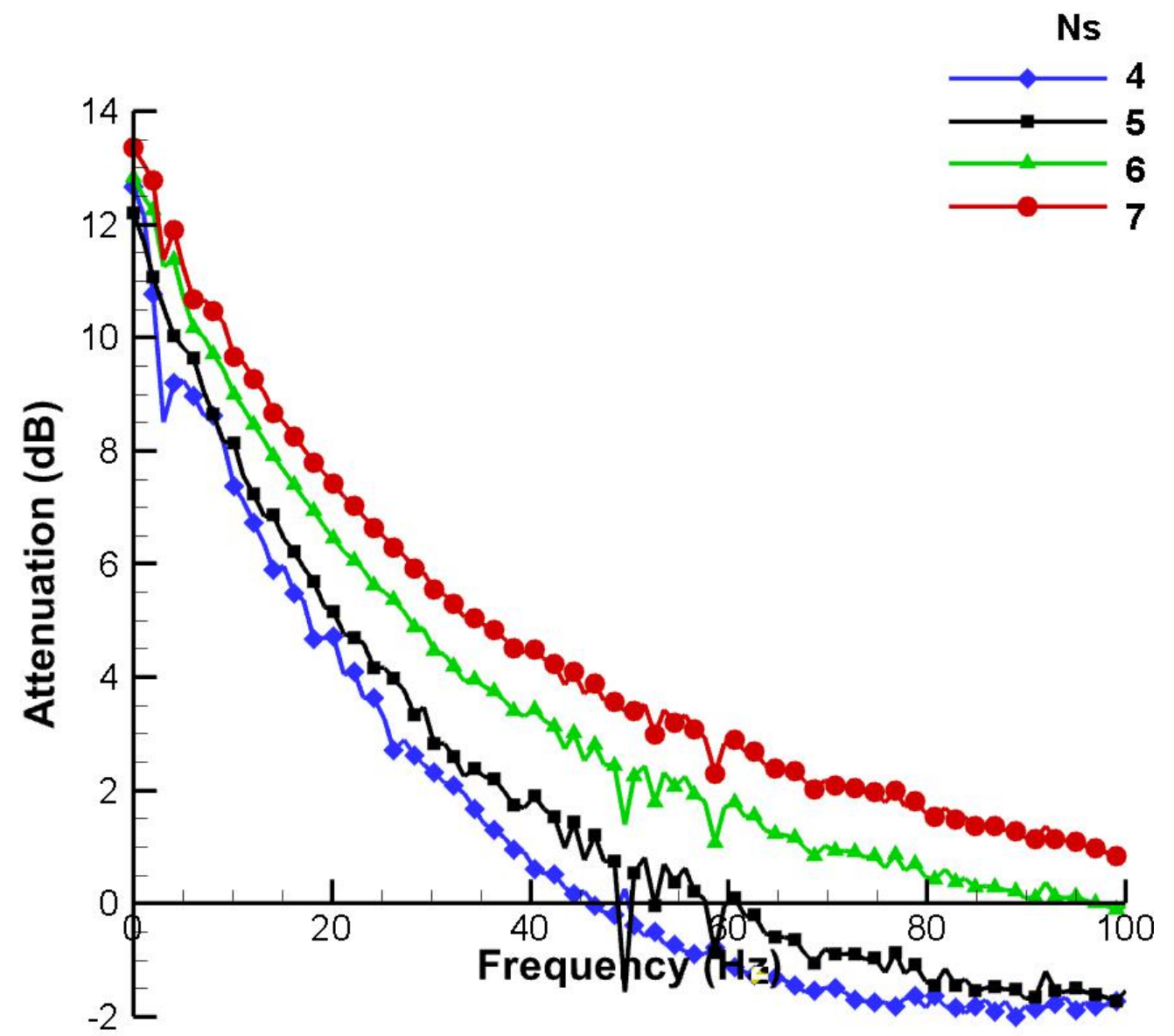

FIG. 11. Effect of the number of sensors $N_{s}$ on attenuation of broadband noise. 


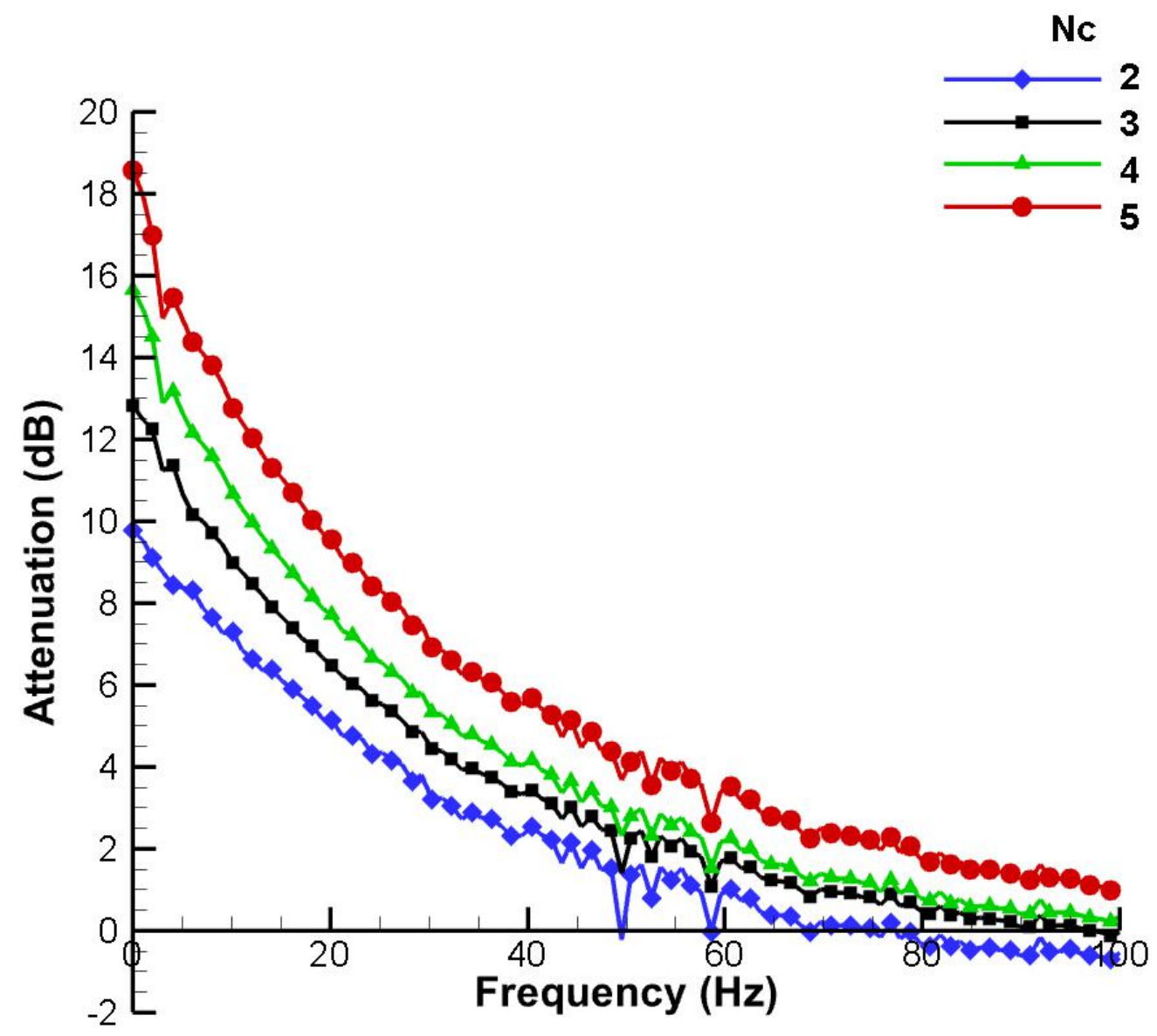

FIG. 12. Effect of the number of controls $N_{c}$ on attenuation of broadband noise. 


\section{CONCLUSION}

The applicability of the nonlocal potential-based algorithm for a real-time noise attenuation has been demonstrated via a series of numerical experiments in the time domain. It is shown that the noise attenuation achieved with a local control deteriorates with the presence of desired sound even if the total primary field at the perimeter of the domain to be shielded is available. This result occurs because a discrete set of controls is not able to provide a strict projection property of the surface potentials. In addition, the real-time ASC becomes unrealistic with the local control if a desired sound component is present and to be retained. In this case the noise level growths with time because of the reverse effect of the discrete control. As has been shown, the nonlocal control enables us to retain a significant noise attenuation even if the input field from the primary sources is unavailable and limited only to the total field from the primary and secondary sources. It turns out that with the nonlocal control the level of noise attenuation is mostly determined by the undesired incident field and weakly depends on the desired interior sound with the same level of intensity. This property is not realized with the local control.

The sensitivity analysis demonstrates that the density of sensors generally plays a more important role than the density of controls in the case of nonlocal control. On average, it is required to double the density of controls with respect to the density of sensors. In practical applications, the Hausdorff distance between the surfaces with sensors and controls is not allowed to be much less than the minimal wavelength to be attenuated. As demonstrated on different test cases including a broadband noise, for low enough frequencies the nonlocal 
${ }_{473}$ control provides practically the same level of noise attenuation regardless the presence of 474 desired sound.

475 VII. ACKNOWLEDGEMENT

476 The authors are grateful to the referees for useful remarks which essentially improved the 477 quality of the paper. 
Bai, M. R., and Chen, H. (2000). "Active cancellation of noise in a car cabin using the zero spillover controller," Journal of sound and vibration 235(5), 787-800.

Baker, B. B., and Copson, E. (1939). "The mathematical theory of hyugens' principle," mthp .

Broadbent, E. (1976). "Noise shielding for aircraft," Progress in Aerospace Sciences 17, 231-268.

Canevet, G. (1978). "Active sound absorption in an air conditioning duct," JSV 58(3), 333-345.

Elliott, S. (2001). "Signal processing for active control" .

Elliott, S., and Nelson, P. (1990). "The active control of sound," Electronics \& communication engineering journal 2(4), 127-136.

Elliott, S. J., and Sutton, T. J. (1996). "Performance of feedforward and feedback systems for active control," IEEE Transactions on Speech and Audio Processing 4(3), 214-223.

Epain, N., and Friot, E. (2007). "Active control of sound inside a sphere via control of the acoustic pressure at the boundary surface," J Sound and Vibration 299(3), 587-604.

Hansen, C., and Snyder, S. (1996). Active Control of Noise and Vibration (CRC Press).

Hansen, C., Snyder, S., Qiu, X., Brooks, L., and Moreau, D. (2012). Active control of noise and vibration (CRC press).

Huang, H., Qiu, X., and J, K. (2011). "Active noise attenuation in ventilation windows. part ii. feedback control," JASA 130, 176-188. 
Jakob, A., and Moser, M. (2003a). "Active control of double-glazed windows. part i. feedforward control," Applied Acoustics 64, 163-82.

Jakob, A., and Moser, M. (2003b). "Active control of double-glazed windows. part ii. feedback control," Applied Acoustics 64, 183-96.

Kletschkowski, T. (2011). Adaptive feed-forward control of low frequency interior noise, $\mathbf{5 6}$ (Springer Science \& Business Media).

Konyaev, S., Lebedev, V., and Fedoryuk, M. (1977). "Discrete approximation of a spherical huygens surface," Sov. Phys. Acoust. 23(4), 373-374.

Kwon, B., and Park, Y. (2011). "Active window based on the prediction of interior sound field: experiment for a band-limited noise," Inter-Noise 2011, Osaka, Japan, September $4-7$.

Landaluze, J., Portilla, I., Pagalday, J., Martinez, A., and Reyero, R. (2003). "Application of active noise control to an elevator cabin," Control engineering practice 11(12), 14231431.

Lim, H., Utyuzhnikov, S., Lam, Y., and Kelly, L. (2014). "Potential-based methodology for active sound control in three dimensional settings," J Acoustical Society of America 136(3), 1101-1111.

Lim, H., Utyuzhnikov, S., Lam, Y., and Turan, A. (2011). "Multi-domain active sound control and noise shielding," J Acoustical Society of America 129(2), 717-725.

Lim, H., Utyuzhnikov, S., Lam, Y., Turan, A., Avis, M., Ryaben'Kii, V., and Tsynkov, S. (2009). "Experimental validation of the active noise control methodology based on difference potentials," AIAA J 47(4), 874-884. 
Loncaric, J., Ryaben'kii, V., and Tsynkov, S. (2001). "Active shielding and control of noise," SIAM J Applied Mathematics 62(2), 563-596.

Malyuzhinets, G. (1971). "An unsteady diffraction problem for the wave equation with compactly supported right-hand side," Proceedings of the Acoustics Institute, USSR Academy of Sciences 124-139.

Nelson, P. A., and Elliott, S. J. (1991). Active control of sound (Academic press).

Ntumy, E. A., and Utyuzhnikov, S. V. (2014). "Active sound control in 3D bounded regions," Wave Motion 51(2), 284-295.

Ntumy, E. A., and Utyuzhnikov, S. V. (2015). "Active sound control in composite regions," Applied Numerical Mathematics 93, 242-253.

Petropavlovsky, S., Tsynkov, S., and Turkel, E. (2018). "A method of boundary equations for unsteady hyperbolic problems in 3d," J. Computational Physics 365, 294-323.

Ryabenkii, V. (1995). "A difference shielding problem," Funct. Anal. Appl 29, 70-71.

Ryabenkii, V. (2002). "Method of difference potentials and its applications.," .

Ryaben'kii, V. (2011). "Model of real-time active noise shielding of a given subdomain subject to external noise sources," J. Comp. Math. and Mathematical Physics 51(3), 444454.

Ryaben'kii, V., Tsynkov, S., and Utyuzhnikov, S. (2007). "Inverse source problem and active shielding for composite domains," Applied Mathematics Letters 20(5), 511-515.

Ryaben'kii, V., and Utyuzhnikov, S. (2006a). "Active shielding model for hyperbolic equations," IMA Journal of Applied Mathematics 924-939. 
Ryaben'kii, V., and Utyuzhnikov, S. (2007). "Differential and finite-difference problems of active shielding," J. Applied Numerical Mathematics 57(4), 374-382.

Ryaben'kii, V. S. (2012). Method of difference potentials and its applications, 30 (Springer Science \& Business Media).

Ryaben'kii, V. S., and Utyuzhnikov, S. V. (2006b). "Active shielding model for hyperbolic equations," IMA J Applied Mathematics 71(6), 924-939.

Sieck, C., and Lau, S. (2011). "Noise propagation through open windows of finite depth into an enclosure," Proceedings of Meetings on Acoustics 11.

Tokhi, M. O., Veres, S., and Veres, S. M. (2002). Active sound and vibration control: theory and applications, 62 (Iet).

Tsynkov, S. V. (2003). "On the definition of surface potentials for finite-difference operators," Journal of scientific computing 18(2), 155-189.

Utyuzhnikov, S. V. (2009a). "Active wave control and generalized surface potentials," Advances in Applied Mathematics 43(2), 101-112.

Utyuzhnikov, S. V. (2009b). "Generalized Calderón-Ryaben'kii's potentials," IMA J Applied Mathematics 74(1), 128-148.

Utyuzhnikov, S. V. (2010a). "Non-stationary problem of active sound control in bounded domains," J Computational and Applied Mathematics 234(6), 1725-1731.

Utyuzhnikov, S. V. (2010b). "Nonlinear problem of active sound control," J Computational and Applied Mathematics 234(1), 215-223.

Utyuzhnikov, S. V. (2014). "Real-time active wave control with preservation of wanted field," IMA J Applied Mathematics 79(6), 1126-1138. 
Utyuzhnikov, S. V. (2017). "A practical algorithm for real-time active sound control with preservation of interior sound," Computers \& Fluids 157, 175-181.

Wang, C., Gao, H., Yu, L., Yan, W., and Xue, Q. (2016). "Portable low-frequency noise reduction device for both small open and closed spaces," Shock and Vibration .

Williams, J. F. (1984). "Review lecture-anti-sound," Proc. R. Soc. Lond. A 395(1808), $63-88$.

Zhou, C., and Utyuzhnikov, S. (2020). "Real-time active noise control with preservation of desired sound," Applied Acoustics 157, 106971.

Zou, H., Lu, J., and Qiu, X. (2010). "The active noise barrier with decentralized feedforward control system," in Proceedings of 17th International Congress on Sound and Vibration. 